\title{
ON THE $L$-STABILIZATION OF THE DOUBLE INTEGRATOR SUBJECT TO INPUT SATURATION
}

\author{
YACINE CHITOUR ${ }^{1}$
}

\begin{abstract}
We consider a finite-dimensional control system $(\Sigma) \dot{x}(t)=f(x(t), u(t))$, such that there exists a feedback stabilizer $k$ that renders $\dot{x}=f(x, k(x))$ globally asymptotically stable. Moreover, for $(H, p, q)$ with $H$ an output map and $1 \leq p \leq q \leq \infty$, we assume that there exists a $\mathcal{K}_{\infty}$-function $\alpha$ such that $\left\|H\left(x_{u}\right)\right\|_{q} \leq \alpha\left(\|u\|_{p}\right)$, where $x_{u}$ is the maximal solution of $(\Sigma)_{k} \dot{x}(t)=f(x(t), k(x(t))+u(t))$, corresponding to $u$ and to the initial condition $x(0)=0$. Then, the gain function $G_{(H, p, q)}$ of $(H, p, q)$ given by$$
G_{(H, p, q)}(X) \stackrel{\text { def }}{=} \sup _{\|u\|_{p}=X}\left\|H\left(x_{u}\right)\right\|_{q},
$$

is well-defined. We call profile of $k$ for $(H, p, q)$ any $\mathcal{K}_{\infty}$-function which is of the same order of magnitude as $G_{(H, p, q)}$. For the double integrator subject to input saturation and stabilized by $k_{L}(x)=-\left(\begin{array}{ll}1 & 1\end{array}\right)^{T} x$, we determine the profiles corresponding to the main output maps. In particular, if $\sigma_{0}$ is used to denote the standard saturation function, we show that the $L_{2}$-gain from the output of the saturation nonlinearity to $u$ of the system $\ddot{x}=\sigma_{0}(-x-\dot{x}+u)$ with $x(0)=\dot{x}(0)=0$, is finite. We also provide a class of feedback stabilizers $k_{F}$ that have a linear profile for $(x, p, p), 1 \leq p \leq \infty$. For instance, we show that the $L_{2}$-gains from $x$ and $\dot{x}$ to $u$ of the system $\ddot{x}=\sigma_{0}\left(-x-\dot{x}-(\dot{x})^{3}+u\right)$ with $x(0)=\dot{x}(0)=0$, are finite.
\end{abstract}

Mathematics Subject Classification. 93D15, 93D21, 93D30.

Received November 22, 1999. Revised January, 2001.

\section{INTRODUCTION}

Let $(\Sigma)$ be the finite dimensional control system of the form:

$$
(\Sigma) \dot{x}(t)=f(x(t), u(t))
$$

where states $x(t) \in \mathbb{R}^{n}$ for all $t \geq 0$ (the integer $n$ is the dimension of $(\Sigma)$ ), inputs $u:[0, \infty) \rightarrow \mathbb{R}^{m}$ are measurable locally essentially bounded maps (the integer $m$ is the dimension of the input space), and $f$ is locally Lipschitz in $(x, u)$ with $f(0,0)=0$. Assume that $(\Sigma)$ admits a feedback stabilizer, i.e., a map $k: \mathbb{R}^{n} \rightarrow \mathbb{R}^{m}$ locally bounded such that $k(0)=0$ and the closed loop system given by

$$
\dot{x}=f(x, k(x)),
$$

Keywords and phrases: Nonlinear control systems, $L^{p}$-stabilization, input-to-state stability, finite-gain stability, input saturation, Lyapunov function.

1 Université Paris XI, Département de Mathématiques, 91405 Orsay, France; e-mail: Yacine.Chitour@math.u-psud.fr 
is globally asymptotically stable (GAS for short) with respect to the origin. For basic terminology on control theory, we refer to [9]. We are interested in the robustness of the feedback $k$, i.e., in the stability of $k$ with respect to measurement and actuator noise. We first recall the following definition: the class of $\mathcal{K}_{\infty}$-functions consists of all $\alpha: \mathbb{R}^{+} \rightarrow \mathbb{R}^{+}$which are continuous, strictly increasing, unbounded and satisfy $\alpha(0)=0$. For simplicity of the exposition, we also assume that $k$ is locally Lipschitz. We hence consider the control system $(\Sigma)_{k}$ given by

$$
(\Sigma)_{k} \quad \dot{x}(t)=f(x(t), k(x(t))+u(t))
$$

We use $x_{u}$ to denote the maximal solution of (1.3) corresponding to $u$ and to the initial condition $x(0)=0$. For $p, q \in[1, \infty], p \leq q$, and an output map $H: \mathbb{R}^{n} \times \mathbb{R}^{m} \rightarrow \mathbb{R}^{l}$ locally Lipschitz with $H(0,0)=0$, we then ask if $(\Sigma)_{k}$ verifies the following properties:

$$
\begin{aligned}
& \left(P_{(H, p, q)}^{1}\right) \text { the assignment } u \mapsto H\left(x_{u}, u\right) \text { defines a map } F_{(H, p, q)}: L^{p}\left(\mathbb{R}^{+}, \mathbb{R}^{m}\right) \rightarrow L^{q}\left(\mathbb{R}^{+}, \mathbb{R}^{l}\right) \\
& \left(P_{(H, p, q)}^{2}\right) \text { there exists } \alpha_{(H, p, q)} \in \mathcal{K}_{\infty} \text { such that }
\end{aligned}
$$

$$
\left\|F_{(H, p, q)}(u)\right\|_{q} \leq \alpha_{(H, p, q)}\left(\|u\|_{p}\right)
$$

(Indices such as $(H, p, q)$ are dropped when the context is clear.) The main output maps $H$ are $x, f(x, u)$, (the integer $l$ is then equal to $n), h^{T} x, h^{T} f(x, u)$ where $h$ is a fixed nonzero vector of $\mathbb{R}^{n}$ (the integer $l$ is then equal to 1). We also have local versions of $\left(P_{(H, p, q)}^{2}\right)$ when (1.4) only holds in a neighborhood of 0 or a neighborhood of $\infty$. The feedback $k$ is said to be of shape $\alpha$ for $(H, p, q)$ (at 0 or at $\infty$ respectively) or $\alpha$ is a shape of $k$ for $(H, p, q)$ (at 0 or at $\infty$ respectively) if $(H, p, q)$ satisfies $\left(P_{(H, p, q)}^{2}\right)$ (in a neighborhood of 0 or $\infty$ respectively) with $C \alpha \in \mathcal{K}_{\infty}$ in equation (1.4) and $C>0$ is a constant independent of $u$. Remark that if there exists a shape $\alpha$ of $k$ for $(H, p, q)$, we can define the gain function $G_{(H, p, q)}: \mathbb{R}^{+} \rightarrow \mathbb{R}^{+}$associated to $(H, p, q)$ by

$$
G_{(H, p, q)}(X) \stackrel{\text { def }}{=} \sup _{\|u\|_{p}=X}\left\|F_{(H, p, q)}(u)\right\|_{q} .
$$

Then $G$ is a nondecreasing function, continuous at 0 with $G(0)=0$. In other words, $k$ is of shape $\alpha$ for $(H, p, q)$ (at 0 or at $\infty$ respectively) if $G_{(H, p, q)}=O(\alpha)$ (at 0 or $\infty$ respectively). We say that $k$ is of profile $\alpha$ for $(H, p, q)$ at 0 (at $\infty$ respectively) or $\alpha$ is a profile of $k$ for $(H, p, q)$ at 0 (at $\infty$ respectively) if there exists $c_{1}, c_{2}, X_{0}>0$ such that for every $X<X_{0}\left(X>X_{0}\right.$ respectively),

$$
c_{1} G_{(H, p, q)}(X) \leq \alpha(X) \leq c_{2} G_{(H, p, q)}(X)
$$

This can be denoted by $\alpha \asymp_{0} G_{(H, p, q)}\left(\alpha \asymp_{\infty} G_{(H, p, q)}\right.$ respectively). When $u \mapsto f(0, u)$ and $u \mapsto H(0, u)$ are not identically zero, and if the gain function $G$ is defined and continuous at 0 , then a profile for $G$ exists at 0 . If, in addition, $G$ is unbounded, then it also exists at $\infty$ (see proof in Lem. 2.2 below). Note that a profile is uniquely determined up to the multiplication by a positive bounded function. A profile of $k$ for $(H, p, q)$ both at 0 and at $\infty$ is simply called a profile of $k$ for $(H, p, q)$.

A natural way to quantify the robustness of a feedback stabilizer $k$ satisfying $\left(P_{(H, p, q)}^{2}\right)$ for some $(H, p, q)$ is to determine (when possible) the asymptotic behaviors of a corresponding profile $\alpha$ when $\|u\|_{p}$ tends firstly to 0 and secondly to $\infty$. Moreover, given two feedback stabilizers $k_{1}$ and $k_{2}$ for $(\Sigma)$ and $(H, p, q)$, we can compare $k_{1}$ and $k_{2}$ by comparing the asymptotic behaviors at 0 and $\infty$ of their respective profiles (when they exist). In particular we say that $k_{1}$ is better than $k_{2}$ at 0 (at $\infty$ respectively) for $(H, p, q)$ if we have, for their corresponding profiles $\alpha_{1}, \alpha_{2}$ (when they exist), that $\alpha_{1}(X)=o\left(\alpha_{2}(X)\right)$ as $X$ tends to 0 (to $\infty$ respectively). Therefore, the notion of profile is a weaker generalization of the concept of finite-gain stability as it is defined in input/output 
studies and related works on computation of norms for nonlinear systems in state-space form $(c f$. [2,15] and the references given therein). The definition of profile is also closely linked to the concept of input-to-state stability (ISS) and related notions (e.g. integral input-to-state stability etc.) see [10]. For instance, if $H(x, u)=x$ and $p=q=\infty$, having a shape for $(x, \infty, \infty)$ is equivalent to have the (ISS) property (cf. Th. $H$ in [10]). To illustrate the previous definitions, we consider a simple case namely the linear one. We assume that $(\Sigma)$ is a linear system $\dot{x}=A x+B u$ such that the uncontrollable modes have negative real parts. Then, there exists a linear feedback $k$ such that for every $p, q \in[1, \infty], p \leq q$ and main output map $H, k$ is of linear profile (i.e. the corresponding $\alpha$ is a linear function for $(H, p, q))$. We can see with the previous example that that the notion of linear profile is less precise than the notion of finite gain.

Under which conditions on $f, k$ and on $(H, p, q)$, the control system $(\Sigma)_{k}$ satisfies the properties $\left(P^{1}\right)_{(H, p, q)}$ or $\left(P^{2}\right)_{(H, p, q)}$ are general issues one can address. In addition, other questions can be considered such as the relationships between the two previous properties and (in the spirit of $I S S$ results) the links between the fact of admitting a profile and admitting a control Lyapunov function satisfying some dissipative inequality. In this paper, we rather focus on a particular control system $\left(S I_{2}\right)$ (defined below) and we address through that example the two following issues: given a control system admitting a feedback stabilizer $k$ and a main output map $(H, p, q)$, then find first the profile of $k$ for $(H, p, q)$ and second, a feedback stabilizer with the best profile for $(H, p, q)$.

The control system $\left(S I_{2}\right)$ belongs to the class of linear control systems subject to input saturation:

$$
\dot{x}=A x+B \sigma(u)
$$

where $A$ is an $n \times n$ matrix, $B$ an $n \times m$ matrix and $\sigma$ is of "saturation" type. For instance, arctan, tanh and the standard saturation function $\sigma_{0}(s)=\frac{s}{\max (1,|s|)}$ are 1-dimensional examples of saturation functions. For this class of systems, examples of feedback stabilizers are well-known under the necessary and sufficient condition that the controllable modes are of non positive real part and the uncontrollable ones of negative real part $(c f$. [8,11-14], and references therein). The interesting problems for control systems (1.6) occur when $A$ admits at least one eigenvalue $\lambda$ with zero real part and more particularly when the multiplicity of $\lambda$ is larger than one (we refer to the latter as the critical case). The feedback stabilizer is necessarily nonlinear in general: in [3] and [14], it is shown that, if $(A, B)$ is controllable, $m=1$ and $A$ is equal to the $n$ th-Jordan matrix, then the corresponding control system $\left(S I_{n}\right)$, called the $n$-th-integrator with saturated input, cannot be globally stabilized by means of a linear feedback if $n \geq 3$. In [5], the authors consider the case when $(A, B)$ is controllable and $A$ is just neutrally stable (i.e. the eigenvalues of $A$ have non positive real parts and there are no nontrivial Jordan blocks). It is shown there, that for the linear feedback stabilizer given by $k(x) \stackrel{\text { def }}{=}-B^{T} x$, we have for every $p \leq q \in[1, \infty)$ or $p=q=\infty$ and $H(x, u)=x$, a linear profile for $(H, p, q)$. Moreover, for $(H, p, q)=(x, p, \infty)$ with $p$ finite, the profile of $k$ is linear at 0 and equal to $X \mapsto X^{p / p+1}$ at $\infty$. Finally, as a first result regarding the critical case, it is shown in [5] that for $\left(S I_{2}\right)$, the linear feedback stabilizer $k_{L}(x) \stackrel{\text { def }}{=}-\left(\begin{array}{ll}1 & 1\end{array}\right)^{T} x$ is not of linear profile at $\infty$ for $(x, p, p)$ with $p \in[1, \infty]$. For related work, in [6], the authors show that any linear system subject to input saturation with poles of non positive real parts can be semi-globally stabilized (that is, on compact sets) by means of linear feedback. In addition, they show in [7] that for every $D>0$, there exists a linear feedback stabilizer which is of linear profile (depending on $D$ ) under the restriction that all the inputs considered are essentially bounded by $D$.

In the present paper, we consider the control system $\left(S I_{2}\right)$ given by

$$
\left(S I_{2}\right)\left\{\begin{array}{l}
\dot{x}_{1}=x_{2}, \\
\dot{x}_{2}=\sigma(u) .
\end{array}\right.
$$

In a first part we determine the profiles of the linear feedback stabilizer $k_{L}$ for the main output maps. We show that, at 0 , all the profiles considered are linear and, at $\infty$, they are of the type $X \mapsto X^{r}$, where $r$ depends on $(H, p, q)$ ( $c f$. Th. 2.5 below). Let us illustrate the aforementioned results. For that purpose, $\left(x_{1}, x_{2}\right)$ is used to denote the trajectory of $\left(S I_{2}\right)_{k_{L}}$ starting at 0 and corresponding to $u \in L^{p}(0, \infty)$. Then, we prove that there 
exist $a_{i}, b_{i}>0$ and $C>0$ such that, for every input $u \in L^{2}(0, \infty)$ with $\|u\|_{2}>C$, we have

$$
\begin{aligned}
\left\|x_{1}\right\|_{2} & \leq a_{1}\|u\|_{2}^{2}, \quad\left\|x_{1}\right\|_{\infty} \leq b_{1}\|u\|_{2}^{\frac{4}{3}}, \\
\left\|x_{2}\right\|_{2} & \leq a_{2}\|u\|_{2}^{\frac{4}{3}}, \quad\left\|x_{2}\right\|_{\infty} \leq b_{2}\|u\|_{2}^{\frac{2}{3}}, \\
\left\|\dot{x}_{2}\right\|_{2} & =\left\|\sigma\left(-x_{1}-x_{2}+u\right)\right\|_{2} \leq a_{3}\|u\|_{2} .
\end{aligned}
$$

We also prove that the exponents of $\|u\|_{2}$ in the above equations are best possible. As a corollary, we partially solve part 2 of Problem 36 as defined in [1]: equation (1.9) shows that the $L_{2}$-gain from the output of the saturation nonlinearity to $u$ of the system $\ddot{x}=\sigma_{0}(-x-\dot{x}+u)$ with $x(0)=\dot{x}(0)=0$ is finite. However, we are not able to compute the value of that $L_{2}$-gain.

If one uses $C^{1}$ feedback stabilizers for $\left(S I_{2}\right)$, the best profile one can get for the output map $H(x, u)=x$, is a linear profile. In the second part of the paper, we exhibit a set of nonlinear feedback stabilizers for $\left(S I_{2}\right)$ such that each of them admits a linear profile for $H$ ( $c f$. Th. 2.8 below). We also provide additional results regarding nonzero initial states and the perturbed control system $\left(P S I_{2}\right)_{k}$ :

$$
\left(P S I_{2}\right)_{k} \begin{cases}\dot{x}_{1} & =x_{2}+v_{1} \\ \dot{x}_{2} & =\sigma(k(x)+u)+v_{2}\end{cases}
$$

with $v=\left(v_{1}, v_{2}\right), v_{1}, v_{2} \in L^{p^{\prime}}(0, \infty), p^{\prime} \geq 1$ and $\|v\|_{\infty} \leq C_{0}$, for some constant $C_{0}$ only depending on $\left(S I_{2}\right)(c f$. Ths. 2.6 and 2.9 below). We conclude this introduction with a remark regarding the techniques used to obtain the results. In [5] and [7], the passivity technique is essentially generalized: for $p \in[1, \infty]$, the main argument of the proofs consists of finding a suitable "storage function" $V_{p}$ and establishing for $V_{p}$ a "dissipation inequality" of the form

$$
\frac{\mathrm{d} V_{p}\left(x_{u}(t)\right)}{\mathrm{d} t} \leq-\left\|x_{u}(t)\right\|^{p}+\lambda_{p}\|u(t)\|^{p}
$$

for some constant $\lambda_{p}>0$. We recall that in [5] a non-smooth $V_{p}$ was needed. For more discussion on passivity, see for instance [4] and [15]. In our situation, we are not able to find such storage functions. We just have at our disposal a control Lyapunov function $V$ (in the case of the feedback $k_{L}$, the saturation function $\sigma$ has to be increasing and $V$ is not even (iISS)-Lyapunov - see [10] -). Therefore the "dissipation inequality" satisfied by $V$ does not lead directly to the desired estimates. However, on a time interval $I=\left[t_{0}, t_{1}\right]$ where the variation of $V$ is positive and "large", we estimate the measures of subsets of $I$ where the input $u$ is "big". We can then bound integrals on $I$ of various quantities in terms of $\int_{I}|u|^{p}$ and get global results.

\section{Notations And Statement of the Results}

In this section we define the class of saturation functions to be considered and state the main results we obtain for $\left(S I_{2}\right)$. In addition, we provide simple remarks.

Definition 2.1. We call $\sigma: \mathbb{R} \rightarrow \mathbb{R}$ a saturation function (or an $S$-function) if there exist two real numbers $0<a \leq K_{\sigma}$ such that for all $t, t^{\prime} \in \mathbb{R}$

(i) $\left|\sigma(t)-\sigma\left(t^{\prime}\right)\right| \leq K_{\sigma} \inf \left(1,\left|t-t^{\prime}\right|\right)$;

(ii) $|\sigma(t)-a t| \leq K_{\sigma} t \sigma(t)$.

A constant $K_{\sigma}$ defined as above is called an $S$-bound for $\sigma$. When the context is clear, we simply use $K$ to denote an $S$-bound. Note that $(i)$ is equivalent to the fact that $\sigma$ is bounded and globally Lipschitz. On the other hand, (ii) is equivalent to

$$
t \sigma(t)>0 \text { for } t \neq 0, \liminf _{|t| \rightarrow \infty}|\sigma(t)|>0, \limsup _{t \rightarrow 0} \frac{\sigma(t)-\sigma^{\prime}(0) t}{t^{2}}<\infty .
$$


We say that $\sigma$ is an $i S$-function if $\sigma$ is an increasing $S$-function. If $m$ is a positive integer, $\sigma$ is an $\mathbb{R}^{m}$-valued $S$-function if $\sigma=\left(\sigma_{1}, \ldots, \sigma_{m}\right)$, where each component $\sigma_{i}$ is an $S$-function and

$$
\sigma(t)=\left(\sigma_{1}(t), \ldots, \sigma_{m}(t)\right)
$$

for $x=\left(x_{1}, \ldots, x_{m}\right)^{T}$. Here, we use $(\cdots)^{T}$ to denote the transpose of $(\cdots)$. Throughout this paper, if $x \in \mathbb{R}^{n}$, we use $\|x\| \stackrel{\text { def }}{=}\left(\sum_{i=1}^{n} x_{i}^{2}\right)^{1 / 2}$ to denote the usual Euclidean norm. If $E$ is a measurable set of $\mathbb{R},|E|$ is its Lebesgue measure. If $S$ is a finite set, we use $S^{\#}$ to denote its cardinality. For $p \in[1, \infty]$ and $T>0$, we use $L^{p}$ to denote $L^{p}(0, \infty)$ and we let $\|x\|_{p}\left(\|x\|_{p,[0, T]}\right.$ respectively $)$ denote the $L^{p}$-norm:

$$
\|x\|_{p} \stackrel{\text { def }}{=}\left(\int_{0}^{\infty}\|x(t)\|^{p} \mathrm{~d} t\right)^{1 / p},\left(\|x\|_{p,[0, T]} \stackrel{\text { def }}{=}\left(\int_{0}^{T}\|x(t)\|^{p} \mathrm{~d} t\right)^{1 / p} \text { respectively }\right),
$$

and

$$
\|x\|_{\infty} \stackrel{\text { def }}{=} \text { ess } \sup _{0 \leq t<\infty}\|x(t)\|,\left(\|x\|_{\infty,[0, T]} \stackrel{\text { def }}{=} \text { ess } \sup _{0 \leq t \leq T}\|x(t)\| \text { respectively }\right) .
$$

Various constants depending only on $\left(S I_{2}\right)$ and $(H, p, q)$ will be considered. We will use the symbol const. to denote those that depend on $\left(S I_{2}\right)$ and $(H, p, q)$. Sometimes, a constant term may depend on an extra parameter $R$. In this case, instead of const., we use const. $R$. For $a \in \mathbb{R}$, let $a^{+} \stackrel{\text { def }}{=} \sup (a, 0)$ and we use sgn $a$ to denote the sign of $a\left(\frac{a}{|a|}\right.$ if $a \neq 0$ and 0 otherwise $)$. For $a, b>0$, the symbol $a \ll b$ means that $b$ is much larger than $a$. If $a, b \in \mathbb{R}$, we use $\left.\Delta f\right|_{a} ^{b}$ or $\left.\Delta f\right|_{I}$ to denote $f(b)-f(a)$ where $f$ is a function on $\mathbb{R}$ and $I$ is the interval between $a$ and $b$.

Let us consider a control system of the form $\left(\Sigma_{1}\right)$ together with a feedback stabilizer $k$ that gives rise to a control system of the form $\left(\Sigma_{1}\right)_{k}$. For $(H, p, q)$ with $H$ a main output map and $1 \leq p \leq q \leq \infty$, assume that $\left(P_{(H, p, q)}^{1}\right)$ holds. Suppose that there exists $X_{0}>0$ such that $\sup _{\|u\|_{p}=X_{0}}\left\|F_{(H, p, q)}(u)\right\|_{q}$ is infinite. In this case, we say that $k$ has infinite gain for $(H, p, q)$. Otherwise, the gain function $G_{(H, p, q)}: \mathbb{R}^{+} \rightarrow \mathbb{R}^{+}$is well-defined and we say that $k$ has finite gain $G_{(H, p, q)}$ (or simply $G$ ) for $(H, p, q)$. The function $G$ is not necessarily continuous but we have:

Lemma 2.2. Let $\left(\Sigma_{1}\right)_{k}$ and $(H, p, q)$ be defined as above. Suppose that $k$ has finite gain $G$ for $(H, p, q)$. Then,

(a) $G(X)=\sup _{\|u\|_{p} \leq X}\left\|F_{(H, p, q)}(u)\right\|_{q}$ and thus $G$ is nondecreasing;

(b) the gain function $G: \mathbb{R}^{+} \rightarrow \mathbb{R}^{+}$is well-defined and continuous at 0 if and only if $\left(P_{(H, p, q)}^{2}\right)$ holds. In addition, the shape $\alpha$ given by (1.4) can be taken as a profile of $k$ for $(H, p, q)$ if,

(b1) for $p<\infty, G$ is unbounded;

(b2) for $p=\infty, G$ is unbounded and $\left\|f\left(0, u_{n}\right)\right\|>0$ for some sequence $\left(u_{n}\right)_{n \geq 0}, u_{n} \in \mathbb{R}^{m}$, such that $\lim _{n \rightarrow \infty} u_{n}=0$.

Proof of Lemma 2.2. We assume that $X>0$. Let $A=G(X)$ and $B=\sup _{\|u\|_{p} \leq X}\left\|F_{(H, p, q)}(u)\right\|_{q}$. We clearly have $A \leq B \leq \infty$. Assume first that $B=\infty$. Then, for every $R>0$, there exists $u_{R} \in L^{p}$ such that $\left\|u_{R}\right\|_{p} \leq X$ and $2 R<\left\|F_{(H, p, q)}\left(u_{R}\right)\right\|_{q}<\infty$. If $\left\|u_{R}\right\|_{p}<X$, we change $u_{R}$ outside an interval $\left(0, T_{R}\right)$ in order to construct some $v_{R} \in L^{p}$ such that $\left\|v_{R}\right\|_{p}=X$ and $\left\|F_{(H, p, q)}\left(v_{R}\right)\right\|_{q} \geq R$. The time $T_{R}$ is chosen as follows: if $q$ is finite, we should have $\int_{0}^{T_{R}}\left|F_{(H, p, q)}\left(u_{R}\right)\right|^{q} \geq R^{q}$ and if $q=\infty,\left|F_{(H, p, q)}\left(u_{R}\right)\left(T_{R}\right)\right|=R$. Then, $A \geq R$ for every $R>0$, which implies that $A=\infty$. We now assume that $B$ is finite. For every $\varepsilon>0$, there exists $u_{\varepsilon} \in L^{p}$ such that $\left\|u_{\varepsilon}\right\|_{p} \leq X$ and $B-\varepsilon<\left\|F_{(H, p, q)}\left(u_{\varepsilon}\right)\right\|_{q} \leq B$. Exactly as before, if $\left\|u_{\varepsilon}\right\|_{p}<X$, we change $u_{\varepsilon}$ outside an interval $\left(0, T_{\varepsilon}\right)$ in order to construct some $v_{\varepsilon} \in L^{p}$ such that $\left\|v_{\varepsilon}\right\|_{p}=X$ and $B-2 \varepsilon \leq\left\|F_{(H, p, q)}\left(v_{\varepsilon}\right)\right\|_{q}$. Then $A \geq B-2 \varepsilon$ for every $\varepsilon>0$, which implies that $A \geq B$. 
We now prove item $(b)$. We have two alternatives; either $u \mapsto f(0, u)$ is the zero function or there exists $X_{0} \geq 0$ such that $G(X)>0$ if $X>X_{0}$ and $G\left(X_{0}\right)=0$. In the first case, $x_{u} \equiv 0$ for every locally essentially bounded measurable $u$. Any $\alpha \in \mathcal{K}_{\infty}$ is a shape of $k$ for $(H, p, q)$. In the second case, let $G^{+}\left(X_{0}\right) \stackrel{\text { def }}{=} \lim _{X \rightarrow X_{0}^{+}} G(X)$. If $G^{+}\left(X_{0}\right)>0$, replace $G$ by the function defined by $G$ on $\left(X_{0}, \infty\right)$ and $X \mapsto G^{+}\left(X_{0}\right) \frac{X}{X_{0}}$ on $\left[0, X_{0}\right]$. We can then assume without loss of generality that $G$ is continuous at $X_{0}$. Let $g \stackrel{\text { def }}{=} G\left(X_{0}+1\right)$. The argument that follows is standard. Since $G\left(X_{0}\right)=0$ and $G$ is increasing, we can find a sequence of times $\left(t_{n}\right)_{n \geq 0}$ such that $t_{0}=X_{0}+1$, $G\left(t_{n}\right)=g / 2^{n}$ and for $n \geq 0, G\left(t_{n+1}\right) \leq G(t) \leq G\left(t_{n}\right)$ for $t \in\left[t_{n+1}, t_{n}\right]$. Then $\left(t_{n}\right)_{n \geq 0}$ is a decreasing sequence to $X_{0}$. Let $\alpha$ be the piecewise linear function defined on $\left[X_{0}, X_{0}+1\right]$ such that for $n \geq 0$ and $t \in\left[t_{n+1}, t_{n}\right]$, $\alpha$ is the line segment between $\left(t_{n+1}, 2 G\left(t_{n+1}\right)\right)$ and $\left(t_{n}, 2 G\left(t_{n}\right)\right)$. Then $\alpha$ is continuous, increasing, $\alpha\left(X_{0}\right)=0$ and for all $t \in\left[X_{0}, X_{0}+1\right]$ we have $\frac{\alpha(t)}{4} \leq G(t) \leq \alpha(t)$. If $p<\infty$, then $X_{0}=0$ since there exist $u \in L^{p}$ with arbitrary small $L^{p}$-norm and arbitrary large $L^{\infty}$-norm. If $p=\infty$, take $\bar{\alpha}(t) \stackrel{\text { def }}{=} t+\alpha(t)$. Then, for every $p \in[1, \infty]$, we have constructed a shape of $k$ at 0 for $(H, p, q)$. Remark that the extra hypothesis on $f$ in $(b 2)$ implies that we have $X_{0}=0$.

For the behavior at $\infty$, we distinguish whether $G$ is unbounded or not. When $G$ is unbounded, the procedure described as above holds in a similar way, by considering now a sequence of times $\left(s_{n}\right)_{n \geq 0}$ such that $s_{0}=1$, $G\left(s_{n}\right)=2^{n} g$ and, for $n \geq 0, G\left(s_{n}\right) \leq G(t) \leq G\left(s_{n+1}\right)$ for $t \in\left[s_{n}, s_{n+1}\right]$. It is clear that the function $\alpha$ previously defined is a $\mathcal{K}_{\infty}$-function and is a profile of $k$ for $(H, p, q)$ at $\infty$. If $G$ is bounded, there exists $n_{0}$ such that $2^{n_{0}} g \leq \sup _{t \in \mathbb{R}^{+}} G(t)<2^{n_{0}+1} g$. Then for $t \geq s_{n_{0}+1}$, we take for $\alpha$ a half-line of positive slope starting at $\left(s\left(t_{n_{0}+1}\right), 2 G\left(s_{n_{0}+1}\right)\right)$.

We give next a criterion for a shape to be a profile. This result will be used repeatedly in the sequel.

Lemma 2.3. Let $\left(\Sigma_{1}\right)_{k}$ and $(H, p, q)$ be defined as above. Suppose that $\alpha$ is a shape of $k$ for $(H, p, q)$ such that there exists $K>1$ and

(a) there exist $r>0, X_{0}>1$ and $A \geq 1$ such that we have $\alpha(X / K) \geq \alpha(X) / A K^{r}$ for $X \leq 1 / X_{0}(\alpha(K X) \leq$ $A K^{r} \alpha(X)$ for $X \geq X_{0}$ respectively);

(b) there exist $a>0$ and a sequence $\left(u_{n}\right)_{n \geq 0}\left(\left(v_{n}\right)_{n \geq 0}\right.$ respectively) such that $\left\|u_{n}\right\|_{p} \rightarrow 0,\left\|u_{n+1}\right\|_{p} \geq\left\|u_{n}\right\|_{p} / K$ $\left(\left\|v_{n}\right\|_{p} \rightarrow \infty,\left\|v_{n+1}\right\|_{p} \leq K\left\|v_{n}\right\|_{p}\right)$ and

$$
\frac{\left\|F\left(u_{n}\right)\right\|_{q}}{\alpha\left(\left\|u_{n}\right\|_{p}\right)} \geq a \quad\left(\frac{\left\|F\left(v_{n}\right)\right\|_{q}}{\alpha\left(\left\|v_{n}\right\|_{p}\right)} \geq a \text { respectively }\right)
$$

Then $\alpha$ is a profile of $k$ for $(H, p, q)$ at 0 (at $\infty$ respectively).

Condition (a) simply says that if $\beta(X) \stackrel{\text { def }}{=} \frac{\alpha(X)}{X^{r}}$, then $\beta(K X) / \beta(X)$ is bounded below (above respectively) for $X$ in a neighborhood of 0 (of $\infty$ respectively). This is obvious if $\beta$ is constant.

Proof of Lemma 2.3. We just prove the lemma in the neighborhood of $\infty$. We may also assume that $\left(v_{n}\right)_{n \geq 0}$ is increasing. For $X$ large enough, there exists $n_{0}$ such that $\left\|v_{n_{0}}\right\|_{p} \leq X<\left\|v_{n_{0}+1}\right\|_{p}$. Then, we get

$$
\frac{G(X)}{\alpha(X)} \geq \frac{G\left(\left\|v_{n_{0}}\right\|_{p}\right)}{\alpha\left(\left\|v_{n_{0}}\right\|_{p}\right)} \frac{\alpha\left(\left\|v_{n_{0}}\right\|_{p}\right)}{\alpha(X)} \geq \frac{1}{A K^{r}} \frac{F\left(\left\|v_{n_{0}}\right\|_{p}\right)}{\alpha\left(\left\|v_{n_{0}}\right\|_{p}\right)} \frac{A K^{r} \alpha\left(\left\|v_{n_{0}}\right\|_{p}\right)}{\alpha\left(\left\|v_{n_{0}+1}\right\|_{p}\right)} \geq \frac{a}{A K^{r}}
$$

At the light of Lemma 2.3, one can follow the two-step strategy described below in order to show that the profile of $k$ for $(H, p, q)$ is a certain function $\alpha \in \mathcal{K}$ :

Step 1: Establish that some $\alpha \in \mathcal{K}_{\infty}$ verifies the inequality (1.4).

Step 2: Exhibit a sequence of inputs such that the hypothesis of Lemma 2.3 are satisfied.

Remark 2.4. Let $\left(\Sigma_{1}\right)_{k}$ and $(H, p, q)$ be defined as above, with $H$ a main output map and $q$ finite. Suppose that $(x, u) \mapsto f(x, k(x)+u)$ is differentiable at $(0,0)$ and $k$ has finite gain for $(H, p, q)$. If $\dot{x}=A x+B u$ is the 
linearized control system at $(0,0)$, assume for simplicity that $A$ is Hurwitz. Then, a profile of $k$ at 0 is linear. In addition, there exist $\gamma>0$ and a sequence $\left(u_{n}\right)_{n \geq 0}$ with $\left\|u_{n}\right\|_{p} \rightarrow \infty$ such that

$$
\frac{\left\|F\left(u_{n}\right)\right\|_{q}}{\left\|u_{n}\right\|_{p}} \geq \gamma
$$

(Simply consider inputs of small $L^{\infty}$-norm but large $L^{p}$-norm. The corresponding trajectories have small $L^{\infty}$ norm.) Then, $G$ is unbounded and, if $k$ is of profile $\alpha$ at $\infty$, we have $X=O(\alpha(X))$. Therefore, the real issue is to determine $\alpha$ and the best profile of $k$ for $(H, p, q)$ at $\infty$ is at least linear.

We now state the main results of the paper. In Section 3, we study the linear feedback $k_{L}=-\left(x_{1}+x_{2}\right)$ for $\left(S_{2}\right)$ and get the following two theorems:

Theorem 2.5. Let us consider the control system $\left(S I_{2}\right)_{k_{L}}$, where $\sigma$ is an $(i S)$-function. For $(H, p, q)$ with $H$ a main output map and $p \leq q \in[1, \infty]$, the profile of $k_{L}$ at 0 is linear and, at $\infty$, is as follows:

(1) for $H(x, u)=x$ or $x_{1}, X \mapsto X^{r_{1}(p, q)}$ with $r_{1}(p, q)=\frac{2 p(q+1)}{q(p+1)}$;

(2) for $H(x, u)=x_{2}, X \mapsto X^{r_{2}(p, q)}$ with $r_{2}(p, q)=\frac{p(q+2)}{q(p+1)}$;

(3) for $H(x, u)=\sigma\left(-x_{1}-x_{2}+u\right)=\dot{x}_{2}, X \mapsto X^{p / q}$ with $q<\infty$.

In the previous results, we define $r_{i}(p, \infty)$ and $r_{i}(\infty, \infty)$ as

$$
r_{i}(p, \infty) \stackrel{\text { def }}{=} \lim _{q \rightarrow \infty} r_{i}(p, q) \text { if } p \text { finite and } r_{i}(\infty, \infty) \stackrel{\text { def }}{=} \lim _{p \rightarrow \infty} r_{i}(p, \infty) .
$$

In order to state results relative to $\left(P S I_{2}\right)_{k_{L}}$, we consider the following Lyapunov function for $\left(S I_{2}\right)$ (introduced in [14]):

$$
V\left(x_{1}, x_{2}\right)=x_{2}^{2}+\int_{0}^{x_{1}} \sigma(s) \mathrm{d} s+\int_{0}^{x_{1}+x_{2}} \sigma(s) \mathrm{d} s .
$$

If $\sigma$ is an $(i S)$-function, then $V$ is a strict Lyapunov function for the closed loop system (1.2) associated to $\left(S I_{2}\right)_{k_{L}}$. In addition, $V$ is not a $(i I S S)$-Lyapunov function for the system $\left(S I_{2}\right)_{k_{L}}$. Note though that $V^{\frac{1}{2}}$ is a $(i I S S)$ Lyapunov (but not $(I S S)$ ) for the system $\left(S I_{2}\right)_{k_{L}}$. We have:

Theorem 2.6. Let us consider $\left(P S I_{2}\right)_{k_{L}}$. There exists $\varepsilon_{0}>0$ such that for $p \in[1, \infty], p_{1} \leq p$ and $p_{1}$ finite, $u \in L^{p}, v \in L^{p_{1}}$ and $\|v\|_{\infty} \leq \varepsilon_{0}, \bar{x} \in \mathbb{R}^{2}$, if $\left(x_{1}, x_{2}\right)$ is the trajectory of $\left(P S I_{2}\right)_{k_{L}}$ corresponding to $(u, v)$ starting at $\bar{x}$, we get

(1) for $p_{1} \in[1,2)$,

$$
\begin{gathered}
\left\|x_{1}\right\|_{p} \leq \text { const. }\left(L(v, u, \bar{x})+\|u\|_{p}^{2}+\|v\|_{p_{1}^{p}}^{\frac{p+1}{p} c_{p_{1}}}+V(\bar{x})^{\frac{p+1}{p}}\right), \\
\left\|x_{2}\right\|_{p} \leq \text { const. }\left(L(v, u, \bar{x})+\|u\|_{p}^{\frac{p+2}{p+1}}+\|v\|_{p_{1}^{2 p}}^{\frac{p+2}{2 p} c_{p_{1}}}+V(\bar{x})^{\frac{p+2}{2 p}}\right), \\
\sup \left(\left\|x_{2}\right\|_{\infty},\left\|x_{2}\right\|_{\infty}^{2}\right) \leq \text { const. }\left\|x_{1}\right\|_{\infty} \leq \text { const. }\left(L(v, u, \bar{x})+\|u\|_{p}^{\frac{2 p}{p+1}}+\|v\|_{p_{1}}^{c_{p_{1}}}+V(\bar{x})\right), \\
\left\|\dot{x}_{2}\right\|_{p} \leq \text { const. }\left(L(v, u, \bar{x})+\|v\|_{p_{1}}^{\frac{c_{p_{1}}}{2 p}}+V(\bar{x})^{\frac{p+2}{2 p}}\right),
\end{gathered}
$$

with $L(v, u, \bar{x}) \stackrel{\text { def }}{=}\|v\|_{p_{1}}+\|u\|_{p}+V(\bar{x})^{1 / 2}$ and $c_{p_{1}}=\frac{2 p_{1}}{2-p_{1}}$; 
(2) for $p_{1}=2$, set $Q(v, u, \bar{x}) \stackrel{\text { def }}{=}\left(\|u\|_{p}^{\frac{2 p}{p+1}}+V(\bar{x})+1\right) \mathrm{e}^{\text {const. }\|v\|_{2}^{2}}-1$. We have

$$
\begin{gathered}
\left\|x_{1}\right\|_{p} \leq \text { const. }\left(L(v, u, \bar{x})+\|u\|_{p}^{2}+V(\bar{x})^{\frac{p+1}{p}}+Q(v, u, \bar{x})^{\frac{p+1}{p}}\|v\|_{2}^{\frac{2}{p}}\right), \\
\left\|x_{2}\right\|_{p} \leq \text { const. }\left(L(v, u, \bar{x})+\|u\|_{p}^{\frac{p+2}{p+1}}+V(\bar{x})^{\frac{p+2}{2 p}}+Q(v, u, \bar{x})^{\frac{p+2}{2 p}}\|v\|_{2}^{\frac{2}{p}}\right), \\
\sup \left(\left\|x_{2}\right\|_{\infty},\left\|x_{2}\right\|_{\infty}^{2}\right) \leq \text { const. }\left\|x_{1}\right\|_{\infty} \leq \text { const. }(L(v, u, \bar{x})+Q(v, u, \bar{x})), \\
\left\|\dot{x}_{2}\right\|_{p} \leq \text { const. }\left(L(v, u, \bar{x})+V(\bar{x})^{\frac{p+1}{2 p}}+\left(Q(v, u, \bar{x})\|v\|_{2}\right)^{\frac{2}{p}}\right) ;
\end{gathered}
$$

(3) for $p_{1}>2$ and $\varepsilon>0$, there exist $u, v$ with $u$ continuous and compactly supported, $v \in L^{p_{1}}$ and $\|v\|_{\infty} \leq \varepsilon$, such that the trajectory of $\left(P S I_{2}\right)_{k_{L}}$ corresponding to $(u, v)$ starting at $(0,0)$ is unbounded.

In the statement of Theorem 2.6, the exponents corresponding to the case $p=\infty$ are the limits of the exponents when $p$ tends to $\infty$.

In Section 4, we consider nonlinear feedbacks for $\left(S I_{2}\right)$. For that purpose, we define the class of $\mathcal{F}$-functions as follows:

Definition 2.7. A function $F: \mathbb{R} \rightarrow \mathbb{R}$ is an $\mathcal{F}$-function if $F$ is $C^{1}$, odd, $F^{\prime}(0)=0$ and there exist $r \geq 1$ such that, for $\left|x_{2}\right| \geq 1$, we have

$$
F^{\prime}\left(x_{2}\right) \geq \sup \left(3\left|x_{2}\right|, r \frac{F\left(x_{2}\right)}{x_{2}}\right)
$$

The next theorems describe the performances of the feedback $k_{F}$ defined below:

Theorem 2.8. Let us consider the control system $\left(S I_{2}\right)$ with $\sigma$ an $S$-function, and the feedback $k_{F}(x)$ given by

$$
k_{F}(x) \stackrel{\text { def }}{=}-\left(x_{1}+x_{2}+F\left(x_{2}\right)\right)
$$

where $F$ an $\mathcal{F}$-function. For $p \in[1, \infty], k_{F}$ is a feedback stabilizer for $\left(S I_{2}\right)$ and is of linear profile for $(x, p, p)$.

For instance $-\left(x_{1}+x_{2}+3 x_{2}\left|x_{2}\right|\right)$ and $-\left(x_{1}+x_{2}+x_{2}^{3}\right)$ are examples of $k_{F}$-feedbacks. The function $\bar{F}: \mathbb{R} \rightarrow \mathbb{R}$ defined by $\bar{F}\left(x_{2}\right) \stackrel{\text { def }}{=} x_{2}+F\left(x_{2}\right)$ is a $C^{1}$-diffeomorphism and let $K: \mathbb{R} \rightarrow \mathbb{R}$ be defined by $K(y) \stackrel{\text { def }}{=} \bar{F}^{-1}(y)$. In order to state the results for the perturbed system $\left(P S I_{2}\right)_{k_{F}}$, we need to consider a Lyapunov function $V_{F}$ defined in (4.5) below, for which there exist $a, b>0$ such that, for all $\left(x_{1}, x_{2}\right) \in \mathbb{R}^{2}$, we have

$$
a\left(x_{1}^{2}+\bar{F}\left(x_{2}\right)^{2}\right) \leq V_{F}\left(x_{1}, x_{2}\right) \leq b\left(x_{1}^{2}+\bar{F}\left(x_{2}\right)^{2}\right) .
$$

We get the following theorem (compare to Th. 2 of [5]):

Theorem 2.9. Let us consider $\left(P S I_{2}\right)_{k_{F}}$. There exists $\varepsilon_{0}>0$ such that, for $p \in[1, \infty], u, v \in L^{p}$ and $\|v\|_{\infty} \leq \varepsilon_{0}, \bar{x} \in \mathbb{R}^{2}$, if $\left(x_{1}, x_{2}\right)$ is the trajectory of $\left(P S I_{2}\right)_{k_{F}}$ corresponding to $(u, v)$ starting at $\bar{x}$, the following estimate holds

$$
\|x\|_{p} \leq \text { const. }\left(\|v\|_{p}+\|u\|_{p}+L_{p}\left(V_{F}(\bar{x})^{1 / 2}\right)\right),
$$


where

$$
L_{p}(X) \stackrel{\text { def }}{=}\left(\int_{0}^{X} \frac{s^{p}}{K(s)} \mathrm{d} s\right)^{1 / p} \text { for } p \text { finite and } L_{\infty}(X) \stackrel{\text { def }}{=} X
$$

\section{The linear feedback $k_{L}$}

We rewrite $\left(S I_{2}\right)_{k_{L}}$ as follows

$$
\left\{\begin{array}{l}
\dot{z}=y-\sigma(z+u) \\
\dot{y}=-\sigma(z+u)
\end{array}\right.
$$

where $z=x_{1}+x_{2}, y=x_{2}$ and $\sigma(\cdot)(u(\cdot)$ respectively) stands for $-\sigma(-\cdot)(-u(\cdot)$ respectively). Moreover, up to a time reparameterization and a linear change of variables, we can assume that $\sigma^{\prime}(0)=1$. Let us define $\sigma_{+}=\lim _{+\infty} \sigma, \sigma_{-}=-\lim _{-\infty} \sigma$ and, for $r>0$,

$$
K_{r} \stackrel{\text { def }}{=} \inf \{|\sigma(z)|,|z|>r\} \text { and } \delta_{r} \stackrel{\text { def }}{=} \sup _{|z|,\left|z^{\prime}\right|>\frac{2}{3} r, z z^{\prime}>0}\left|\sigma(z)-\sigma\left(z^{\prime}\right)\right| .
$$

Let $K$ be an $S$-bound for $\sigma$ and $\sigma_{m} \stackrel{\text { def }}{=} \min \left(\sigma_{+}, \sigma_{-}\right), \sigma_{M} \stackrel{\text { def }}{=} \max \left(\sigma_{+}, \sigma_{-}\right)$. Then we have

$$
K \geq \max \left(1, \sigma_{M},\left\|\sigma^{\prime}\right\|_{\infty}\right)
$$

Fix $C, \rho>0$ and $\rho_{p}$ for $p$ finite such that

$$
\begin{aligned}
\rho & \ll 1 \ll C \\
\varepsilon \sigma(\varepsilon C) & >\frac{3}{4} \sigma_{\varepsilon} \text { if } \varepsilon= \pm \\
2 K C \rho_{p} & <\frac{1}{2} \min \left(1, \frac{K_{\rho}^{p}}{2}\right), \\
\rho_{p} & <\frac{\rho}{2} \\
\delta_{C} & \ll \min \left(1, K_{C}, \frac{3}{16} \sigma_{m}\right) .
\end{aligned}
$$

In addition, let $\theta \stackrel{\text { def }}{=} \frac{C}{2(C+K)} \in(0,1 / 2)$. The Lyapunov function defined in $(2.2)$ is written now

$$
V(z, y)=y^{2}+\int_{0}^{z-y} \sigma(s) \mathrm{d} s+\int_{0}^{z} \sigma(s) \mathrm{d} s
$$

For $r>0$, set $V_{r} \stackrel{\text { def }}{=} \max \{V(z, y),\|(z, y)\| \leq r\}$. We use $\dot{V}$ to denote the time derivative of $V$ along trajectories of $\left(S I_{2}\right)_{k_{L}}$ and we have

$$
\dot{V}=-\left.2 y \Delta \sigma\right|_{z} ^{z+u}+\left.y \Delta \sigma\right|_{z} ^{z-y}-\sigma(z) \sigma(z+u)
$$

We sometimes consider a classical Lyapunov function for $\left(S I_{2}\right)$ given by

$$
W(z, y) \stackrel{\text { def }}{=} \frac{y^{2}}{2}+\int_{0}^{z} \sigma(s) \mathrm{d} s
$$


Remark 3.1. When $y z<0, z(z+u) \leq 0$ and $|y| \gg \max (|z|,|u|) \gg 1$, an easy computation shows that $\dot{V} \geq \frac{K}{2}|y|$. This situation explains why $V$ cannot give rise to a dissipation inequality of the kind (1.10). However, in the case described previously, we also have that $|\dot{z}| \geq|y|-K$, which implies that the previous situation firstly cannot last more than a time interval of length 1 and secondly is preceded and followed by time intervals where $|z| \geq|y|$ and whose lengths are of same order as $|y|$. Therefore, by integrating (3.2) on appropriate time intervals, one hopes to achieve the desired results.

Remark 3.2. The above remark is related to the following fact: assume that, at some point $t_{0}$, we have $\left|y\left(t_{0}\right)\right|>K$ and $z\left(t_{0}\right)=0$. Then, $t_{0}$ is an isolated zero of $z$ and, if $t_{1}$ is an another zero of $z$, then

$$
\left|t_{1}-t_{0}\right| \geq 2\left(\frac{\left|y\left(t_{0}\right)\right|}{K}-1\right)
$$

Indeed, without loss of generality, we can assume that $y\left(t_{0}\right)>0$ and $t_{1}>t_{0}$. For $t \geq t_{0}$, we have $y(t) \geq$ $y\left(t_{0}\right)-\left(t-t_{0}\right) K$, which implies that

$$
\dot{z}(t) \geq y\left(t_{0}\right)-K-\left(t-t_{0}\right) K
$$

A simple integration yields equation (3.4).

\subsection{Proof of Theorem $\mathbf{2 . 5}$}

Thanks to Remark 2.4, for the rest of the paper, we look for profiles at $\infty$. Therefore, we can assume that all the inputs considered in the sequel satisfy $\|u\|_{p} \geq C$, for $p \in[1, \infty]$.

The next proposition is a preliminary step towards the proof of Theorem 2.5 for $p=\infty$ and its proof is given in the Appendix:

Proposition 3.3. Let $p \in[1, \infty], T>0$ and $u \in L^{p}$. Let $(z, y)$ be the corresponding trajectory of $\left(P S I_{2}\right)_{k_{L}}$. Then, we have

(a) $\|y\|_{\infty,[0, T]}^{2} \leq$ const. $\|z\|_{\infty,[0, T]}$;

(b) either $\|y\|_{\infty,[0, T]}^{2} \geq \frac{\sigma_{m}}{2}\|z\|_{\infty,[0, T]}$ or

$$
\|z\|_{\infty,[0, T]} \leq \text { const. }\|u\|_{p,[0, T]}^{\frac{2 p}{2 p+1}}
$$

where $\frac{2 p}{2 p+1}$ is taken to be equal to 1 for $p=\infty$.

We now prove (1.4) for $p=\infty$. Taking into account Proposition 3.3, it is enough to show that:

Proposition 3.4. For $u \in L^{\infty}$ and $\|u\|_{\infty} \geq C$ we have

$$
\|z\|_{\infty} \leq \text { const. }\|u\|_{\infty}^{2}
$$

Proof of Proposition 3.4. Let $M=\|u\|_{\infty} \geq C$. For $T>0$, we pick $\left[t_{0}, t_{1}\right] \in[0, T]$ such that we have, for $t \in\left[t_{0}, t_{1}\right]$

$$
W\left(t_{0}\right)=\frac{W_{\max }}{2} \leq W(t) \leq W\left(t_{1}\right)=W_{\max } .
$$

Define $\lambda>0$ such that $W_{\max }=W\left(t_{1}\right)=\lambda M^{2}$. Without loss of generality, we can assume that $\lambda>2$. Then, proving Proposition 3.4 amounts to show that $\lambda$ is actually bounded independently of $u$. This is what we do next. 
Thanks to Proposition 3.3, we can suppose that

$$
\text { const. } \lambda M^{2} \leq y_{\max }^{2} \leq \text { const. } z_{\max } \leq \text { const. } \lambda M^{2},
$$

where $y_{\max }, z_{\max }$ are defined as before.

The conclusion will be the consequence of two lemmas given below and whose proofs are given in the appendix. First, we prove that:

Lemma 3.5. With the previous notations, either $\lambda$ is bounded independently of $u$ or there exists, on $\left[t_{0}, t_{1}\right]$, a sequence of times $\left(t^{j}\right)_{1 \leq j \leq 2 N+1}$ such that

(i) on $\left[t^{1}, t^{2 N+1}\right],|z(t)|=M+C$ exactly for $t$ equal to some $t^{j}, 1 \leq j \leq 2 N+1$;

(ii) $|z(t)| \geq M+C$ on $I_{2 l-1} \stackrel{\text { def }}{=}\left[t^{2 l-1}, t^{2 l}\right]$ and $|z| \leq M+C$ on $I_{2 l}=\left[t^{2 l}, t^{2 l+1}\right]$ for $l=1, \cdots, N$;

(iii) $\left.\Delta W\right|_{t^{1}} ^{t^{2 N+1}} \geq \frac{\lambda}{16} M^{2}$

In a second step, we evaluate the variations of $W$ along $I_{2 l-1}$ and $I_{2 l}$. Set $\left.\left.\Delta W\right|_{j} \stackrel{\text { def }}{=} \Delta W\right|_{t^{j}} ^{t^{j+1}}$, for $j=$ $1, \cdots, 2 N+1$. We get the following key estimate:

Lemma 3.6. With the previous notations, we have for $l=1, \cdots, N$,

$$
\Delta W_{2 l-1}+\Delta W_{2 l} \leq- \text { const. } \sqrt{\lambda} M+\text { const. } M
$$

Finally, by adding all the inequalities (3.7) for $l=1, \cdots, N$, we obtain that

$$
\frac{\lambda}{16} M^{2} \leq\left.\Delta W\right|_{t^{1}} ^{t^{2 N+1}} \leq N M(- \text { const. } \sqrt{\lambda}+\text { const. }) .
$$

Then, $\lambda$ has to be bounded above by some const. and the proof of Proposition 3.4 is finished.

We now consider (3) of Theorem 2.5, in the case where $p=q<\infty$. The first step consists of showing the next proposition:

Proposition 3.7. Let $p \in[1, \infty), u \in L^{p}$ and $x_{u}=(z, y)$ be the trajectory of $\left(S I_{2}\right)_{k_{L}}$ corresponding to $u$ and starting at 0 . Then, we have

$$
\|\sigma(z+u)\|_{p} \leq \text { const. }\|u\|_{p}
$$

Proof of Proposition 3.7. Equation (3.8) will be obtained from the integration of (3.2) on non-overlapping measurable sets $S$, whose countable union is equal to $\mathbb{R}^{+}$. We will distinguish several cases for the $S$ 's and, for each case, we aim at establishing the inequality

$$
\int_{S}\left(\dot{V}+\text { const. }|\sigma(z+u)|^{p}\right) \leq \text { const. } \int_{S}|u|^{p},
$$

either by the corresponding differential inequality $\dot{V}+$ const. $|\sigma(z+u)|^{p} \leq$ const. $|u|^{p}$ or by a direct computation. We will also use repeatedly a result given in [5]:

Remark 3.8. The trajectories of $\left(S I_{2}\right)_{k_{L}}$ corresponding to inputs $u \in L^{p}$ with $p<\infty$ converge to 0 at infinity.

For the rest of the proof, we assume that $\|u\|_{p} \geq C$. We will consider the following cases:

(A) $|y| \leq C$; we have two sub-cases defined by

(A1) $|z| \geq \rho$ : 
(A2) $|z|<\rho$;

(B) $|y|>C$.

Before starting the study of cases $(A)$ and $(B)$, we provide the following remark in order to motivate the technical details of the proof of Proposition 3.7:

Remark 3.9. We have defined the previous cases in such a way that we can reduce the core of the argument to the proof of equation (3.9) when $S=I$, an open interval on which $|z|-|y|-C$ has a constant sign and, more importantly, where $z$ is monotone. As usual, we introduce the subset $E$ of $I$ defined as

$$
E=\left\{t \in I, \quad|u(t)| \geq \frac{|z(t)|}{3} \text { a.e. }\right\}
$$

Next, we write $\dot{V}$ as a sum of several terms (see Eq. (3.14)), whose integrals over $I$ can be easily estimated in terms of $\int_{I}|u|^{p}$ and $|E|$, except for one of them (a quantity $J_{1}$ defined below in Eq. (3.15)). Similarly to the integral $J$ defined in equation (5.13), $J_{1}$ is handled after being written first, by using $z$ as a parameter instead of the time $t$, and finally as a double integral. Note that, in most of the estimations, the hypothesis of $\sigma$ being increasing is crucial.

If $(A 1)$ holds, we have clearly have

$$
\dot{V}+\text { const. }|\sigma(z+u)|^{p} \leq \text { const. }|u|^{p},
$$

whether $|u| \geq \rho_{p}$ or not.

If $(A 2)$ holds, we consider the sets

$$
\begin{aligned}
& S_{0}=\left\{t \geq 0|| y(t) \mid \leq C, V(z(t), y(t))<4 \rho_{p}^{2}\right\} \\
& S_{0}^{\prime}=\{t \geq 0|| y(t)|\leq C,| z(t) \mid<\rho\} .
\end{aligned}
$$

As $\varepsilon$ tends to $0^{+}, S_{0}$ and $S_{0}^{\prime}$ are the limits of the open sets

$$
\begin{aligned}
& S_{\varepsilon}=\left\{t \geq 0|| y(t) \mid<C+\varepsilon, V(z(t), y(t))<4 \rho_{p}^{2}\right\}, \\
& S_{\varepsilon}^{\prime}=\{t \geq 0|| y(t)|<C+\varepsilon,| z(t) \mid<\rho\} .
\end{aligned}
$$

Note that $S_{0} \subset S_{0}^{\prime}$ and $S_{\varepsilon} \subset S_{\varepsilon}^{\prime}$. Thanks to Remark 3.8, the open sets $S_{\varepsilon} / S_{0}$ and $S_{\varepsilon}^{\prime} / S_{0}^{\prime}$ are bounded and their measures tend to zero as $\varepsilon$ tends to $0^{+}$. Since $\dot{V}+$ const. $|\sigma(z+u)|^{p}$ is bounded over $\mathbb{R}^{+}$, in order to establish (3.9) for $E=S_{0}^{\prime}$, it is enough to do it for $E=S_{\varepsilon}^{\prime}$ with constants independent of $\varepsilon$.

On $S_{\varepsilon}$, we have

$$
\dot{V} \leq \text { const. }|u| \sqrt{V}-\text { const. } V \text {. }
$$

Set $V_{p} \stackrel{\text { def }}{=} V^{p / 2}$. Then, using (3.11), we get

$$
\dot{V}_{p} \leq \text { const. } V_{p}^{\frac{p-1}{p}}-\text { const. } V_{p} \text {. }
$$

On each finite interval $\left(t, t^{\prime}\right)$ of $S_{\varepsilon}$, we have $V_{p}(t)=V_{p}\left(t^{\prime}\right)$, which implies that $\left.\Delta V\right|_{t} ^{t^{\prime}}=0$. By integrating (3.12) (and applying Hölder's inequality for $p>1$ ), we get

$$
\left.\Delta V\right|_{t} ^{t^{\prime}}+\text { const. } \int_{t}^{t^{\prime}}|\sigma(z+u)|^{p} \leq \text { const. } \int_{t}^{t^{\prime}}|u|^{p} .
$$


If $(t, \infty) \subset S_{0}$, we have

$$
\int_{t}^{\infty} V_{p}^{p} \leq V_{p}^{p}(t)+\text { const. } \int_{t}^{\infty}|u|^{p}
$$

which leads to (3.13) with $t^{\prime}=\infty$. On $S_{\varepsilon}^{\prime} / S_{\varepsilon}$, we clearly have (3.10) since the dominant term in $\dot{V}$ is $\frac{1}{2} y \sigma(-y)$ if $|u| \leq \rho$. Thus, we have established (3.9) for $E=S_{\varepsilon}^{\prime}$ and $\varepsilon>0$ small enough.

We assume that $(B)|y|>C$ holds. The open set $S_{2}=\{t \geq 0|| y(t) \mid>C\}$ is a union (at most countable) of open intervals $\left(I_{s}\right)$. Since $u \in L^{p}$, the trajectories of $\left(S I_{2}\right)_{k_{L}}$ converge to 0 at infinity, each interval $I_{s}$ has finite length.

We now focus on an interval $I=\left(t_{0}, t_{1}\right)$ where $y\left(t_{0}\right)=y\left(t_{1}\right)=C$ and $y>C$ on $I$. We will prove (3.9) for $S=I$. Let $T \stackrel{\text { def }}{=} t_{1}-t_{0}$. We assume that $T \geq \frac{10 C}{\sigma_{m}}$, otherwise we are back to case $(A)$. We say that condition $(R)$ is satisfied at $t \in I$ if

$$
(R) \quad y(t)+C \leq|z(t)|
$$

Moreover, $z$ is strictly increasing from $I$ to $\left[z_{0}, z_{1}\right]$, where $z_{0}=z\left(t_{0}\right)$ and $z_{1}=z\left(t_{1}\right)$. Then, $z$ is equal to 0 at most once. We have the following situation:

Lemma 3.10. With the notations above, one of the two next cases occurs:

$(B 1) z$ has a constant sign on $I$ and $(R)$ is satisfied on I except (maybe) on an interval $\left(t_{0}^{\prime}, t_{1}^{\prime}\right)$ such that if $z \leq 0, t_{1}^{\prime}=t_{1}$ and there is equality in $(R)$ at $t_{0}^{\prime}$;

if $z \geq 0, t_{0}^{\prime}=t_{0}$ and there is equality in $(R)$ at $t_{1}^{\prime}$;

(B2) z has a unique zero at $\bar{t}$ and $(R)$ is satisfied on $I$, except on an interval $I^{\prime}=\left(t_{0}^{\prime}, t_{1}^{\prime}\right)$ such that $\bar{t} \in I^{\prime}$ and there is equality in $(R)$ at $t_{0}^{\prime}$ and $t_{1}^{\prime}$.

Proof of Lemma 3.10. Consider the function $f$ defined on $I$ by

$$
f(t) \stackrel{\text { def }}{=}|z(t)|-y(t)-C=\operatorname{sgn} z(t) z(t)-y(t)-C
$$

For all $t \in I$, except maybe for at most one point, we have

$$
(\operatorname{sgn} z) \dot{f}=y(t)-(\operatorname{sgn} z(t)+1) \sigma(z(t)+u(t))>0 .
$$

Depending on the sign of $z, f\left(t_{0}\right)$ and $f\left(t_{1}\right)$, we easily get the conclusion.

In each above case, we will establish (3.9) first on $I^{\prime}$ and second on $I / I^{\prime}$. We also need to rewrite (3.2) as

$$
\dot{V}=-\left.2 \dot{z} \Delta \sigma\right|_{z-\theta y} ^{z+u}-\left.\dot{z} \Delta \sigma\right|_{z} ^{z-\theta y}-\left.\dot{z} \Delta \sigma\right|_{z-y} ^{z-\theta y}-\left.\sigma(z+u) \Delta \sigma\right|_{z-y} ^{z+u}-\sigma^{2}(z+u)
$$

We get

$$
\left.\Delta V\right|_{t_{0}} ^{t_{1}}=J_{1}+J_{2}+J_{3}
$$

where

$$
\begin{aligned}
& J_{1}=-\left.\int_{t_{0}}^{t_{1}} \dot{z} \Delta \sigma\right|_{z} ^{z-\theta y}-\left.\int_{t_{0}}^{t_{1}} \dot{z} \Delta \sigma\right|_{z-y} ^{z-\theta y}, \\
& J_{2}=-\int_{t_{0}}^{t_{1}}\left(\left.2 \dot{z} \Delta \sigma\right|_{z-\theta y} ^{z+u}+\left.\sigma(z-y) \Delta \sigma\right|_{z-y} ^{z+u}\right) \\
& J_{3}=-\int_{t_{0}}^{t_{1}}\left(\left[\left.\Delta \sigma\right|_{z-y} ^{z+u}\right]^{2}+\sigma^{2}(z+u)\right) \leq 0 .
\end{aligned}
$$


We now suppose that $(B 1)$ holds. We will only treat the case when $z \geq 0$ and indicate the minor modifications occurring, when $z \leq 0$, before treating case $(B 2)$. Then, we assume that $z \geq 0$ and $I^{\prime}=\left[t_{0}, t_{1}^{\prime}\right)$. Let $T^{\prime}=t_{1}^{\prime}-t_{0}$. We have

$$
T^{\prime} \leq \frac{2}{1-\frac{2 K}{C}},|\Delta V|_{I^{\prime}}\left|\leq C T^{\prime}, \Delta z\right|_{I^{\prime}} \leq C T^{\prime} \text { and }\left.\Delta y\right|_{I^{\prime}} \leq T^{\prime}
$$

by simply taking into account that $\dot{z} \geq C-K$ and $\dot{y} \geq K$. Let us consider the set $E$ given by

$$
E=\left\{t \in I^{\prime},|u(t)|>\frac{z(t)}{3} \text { a.e. }\right\}
$$

Then, we have:

Lemma 3.11. With the previous notations, $|E| \geq$ const. $T^{\prime}$ with const. independent of $C$.

Proof of Lemma 3.11. From (5.8) written on $I^{\prime}$ and the fact that $\left.\Delta y\right|_{I^{\prime}} \geq 0$, we get

$$
0 \leq-\sigma\left(\frac{C}{3}\right)\left(T^{\prime}-|E|-|F|\right)+2 K|E|
$$

where $F=\left\{t \in I^{\prime}, z \leq 1\right\}$. We clearly have

$$
|F| \leq \frac{\frac{C}{3}}{C+\frac{2}{1-\frac{2 K}{C}}} \leq \frac{1}{3}
$$

and, if $F$ is not empty, we have

$$
T^{\prime}-|F| \geq \frac{z\left(t_{1}^{\prime}\right)-\frac{C}{3}}{C+\frac{2}{1-\frac{2 K}{C}}} \geq \frac{5}{4}
$$

We conclude easily.

In the case where $F$ is not empty, $E$ contains a subset $E^{\prime}$ of $E$ of measure $\frac{|E|}{2} \geq$ const. $T^{\prime}$ such that $z \geq$ const. $C$ on $E^{\prime}$. Then $|u|$ will be larger than const. $C$ on $E^{\prime}$ of $E$ and we have

$$
\int_{I^{\prime}}|u|^{p} \geq \int_{E^{\prime}}|u|^{p} \geq \text { const. } C^{p} T^{\prime} \geq|\Delta V|_{I^{\prime}} \mid+ \text { const. } \int_{I^{\prime}}|\sigma(z+u)|^{p} .
$$

In the case where $F$ is empty, we simply take $E^{\prime}=E$, and now

$$
\int_{I^{\prime}}|u|^{p} \geq \int_{E} z \geq \text { const. } \frac{C}{2} T^{\prime} \geq \text { const. } C\left(|\Delta V|_{I^{\prime}} \mid+ \text { const. } \int_{I^{\prime}}|\sigma(z+u)|^{p}\right) .
$$

Then (3.9) is obtained.

We next establish (3.9) on $I / I^{\prime}=\left[t_{1}^{\prime}, t_{1}\right]$. This is a consequence of the following lemma, whose proof is delayed to the Appendix:

Lemma 3.12. Let $J_{1}, J_{2}$ and $J_{3}$ be defined in (3.15, 3.16) and (3.17) respectively. we have

(J1) $J_{1} \leq$ const. $\sup \left(y\left(t_{1}\right), C\right) \delta_{C} \leq$ const. $\delta_{C} T$;

(J2) $J_{2} \leq$ const. $\delta_{C} T+$ const. $\int_{I / I^{\prime}}|u|^{p}$ 
(J3) $J_{3} \leq-$ const. $T+$ const. $\int_{I / I^{\prime}}|u|^{p}$;

where const. is independent of $C$.

Equation (3.9) is obtained on $I$ and that concludes case (B1). When $z \leq 0$, the only differences with the other case occur in the proof of $(J 2)$ given in the Appendix.

We now suppose that case (B2) holds. The case where $z \leq 0$ can be deduced from the case where $z \geq 0$ similarly to what ws done in case $(B 1)$. Then, it is enough to establish (3.9) on the interval $\left[\bar{t}, t_{1}\right]$ where $z$ remains positive. The interval $\left[\bar{t}, t_{1}\right]$ is the union of $I^{\prime}=\left[\bar{t}, t_{1}^{\prime}\right)$ and $\left[t_{1}^{\prime}, t_{1}\right]$. It is easy to see that the procedure that led to $(3.9)$ on $I / I^{\prime}$ above (the inequalities $(3.15,3.16)$ and $(3.17)$ together with Lems. 3.12 and 5.1) will also prove (3.9) on $\left[t_{1}^{\prime}, t_{1}\right]$. Therefore, the last part of the proof of Proposition 3.7 consists of getting (3.9) on $I^{\prime}$.

We can suppose that $y(\bar{t}) \geq 8 C$ since $t_{1}-\bar{t}$ can be taken larger than $\frac{10 C}{\sigma_{m}}$. Indeed, if $y(\bar{t}) \leq 8 C$, we reproduce the argument involving the set $E$, defined in (3.18), and Lemma 3.11 (with $\left[\bar{t}, t_{1}\right]$ now instead of $I^{\prime}$ ) and obtain (3.9).

Until the end of the proof of Proposition 3.7, we assume that $y(\bar{t}) \geq 8 C$.

We have that $z(\bar{t})=0$ and $z\left(t_{1}^{\prime}\right)=y\left(t_{1}^{\prime}\right)+C$. Let

$$
T^{\prime \prime} \stackrel{\text { def }}{=} t_{1}^{\prime}-\bar{t} \text { and } \bar{y} \stackrel{\text { def }}{=} \frac{\int_{\bar{t}}^{t_{1}^{\prime}} y}{T^{\prime \prime}} .
$$

Since

$$
-K \leq \dot{y} \leq K \text { and } T^{\prime \prime} C \leq T^{\prime \prime} \bar{y}=\int_{\bar{t}}^{t_{1}^{\prime}} y=C+y(\bar{t}),
$$

we get $T^{\prime} \leq 1+\frac{y(\bar{t})}{C}$ and $y(\bar{t})-K T^{\prime \prime} \leq \bar{y} \leq y(\bar{t})+K T^{\prime \prime}$. Then, we get

$$
y(\bar{t})\left(1-\frac{2 K}{C}\right) \leq \bar{y} \leq y(\bar{t})\left(1+\frac{2 K}{C}\right) .
$$

We finally obtain the following estimate for $T^{\prime \prime}$ :

$$
\frac{6}{7} \leq 1-\frac{2 K}{C} \leq \frac{1+\frac{C}{y(t)}}{1+\frac{2 K}{C}} \leq T^{\prime \prime} \leq \frac{1+\frac{C}{y(t)}}{1-\frac{2 K}{C}} \leq \frac{9}{8}\left(1+\frac{4 K}{C}\right)
$$

Define $t_{0}^{\prime \prime} \in\left[\bar{t}, t_{1}^{\prime}\right]$ such that $z\left(t_{0}^{\prime \prime}\right)=y\left(t_{0}^{\prime \prime}\right)$. We have

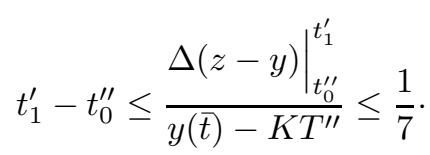

Then, $T_{1} \stackrel{\text { def }}{=} t_{0}^{\prime \prime}-\bar{t} \geq 1-\frac{2 K}{C}-\frac{1}{7} \geq \frac{5}{7}$. Using (3.2), we have

$$
\left.\Delta V\right|_{\bar{t}} ^{t_{1}^{\prime}}+\int_{\bar{t}}^{t_{1}^{\prime}}|\sigma(z+u)|^{p} \leq J+J^{\prime}-\int_{\bar{t}}^{t_{1}^{\prime}} \sigma(z) \sigma(z+u),
$$

where

$$
J \stackrel{\text { def }}{=}-2 \int_{\bar{t}}^{t_{1}^{\prime}} y(\sigma(z+u)-\sigma(z)) \text { and } J^{\prime} \stackrel{\text { def }}{=} \int_{\bar{t}}^{t_{0}^{\prime \prime}} y(\sigma(z-y)-\sigma(z)) .
$$


We first have that

and

$$
-\int_{\bar{t}}^{t_{1}^{\prime}} \sigma(z) \sigma(z+u) \leq 2 K^{2}
$$

$$
J^{\prime} \leq-\int_{\bar{t}}^{t_{0}^{\prime \prime}} y \sigma(z) \leq-\frac{\sigma(1)}{2} T_{1} y(\bar{t}) .
$$

Suppose that $|J| \leq\left|J^{\prime}\right|$. Then (3.9) follows readily. If $|J|>\left|J^{\prime}\right|$, by taking into account the next equation,

$$
|J| \leq 3\left(T^{\prime \prime}-|E|-|F|\right) y(\bar{t}) \delta_{C}+5 K y(\bar{t})|E|,
$$

with $E=\left\{t \in\left[\bar{t}, t_{1}^{\prime}\right],|u|>\frac{z}{3}\right.$ a.e. $\}$ and $F=\left\{t \in\left[\bar{t}, t_{1}^{\prime}\right],|z| \leq 1\right\}$, we obtain, as in Lemma 3.11,

$$
|E| \geq \text { const. } T^{\prime \prime} \text {. }
$$

This implies that (3.9) holds on $\left[\bar{t}, t_{1}^{\prime}\right]$. Then, case $(B)$ is finished, which ends the proof of Proposition 3.7.

The second step of (3) of Theorem 2.5 in the case where $p=q<\infty$ consists of providing an appropriate sequence of inputs $u_{n} \in L^{p}$ satisfying the conditions of Lemma 2.3. This is done in the Appendix.

We now prove (1) and (2) of Theorem 2.5 in the case where $p=q<\infty$. We first establish the next proposition:

Proposition 3.13. Let $p \in[1, \infty), u \in L^{p}$ and $x_{u}=(z, y)$ be the trajectory of $\left(S I_{2}\right)_{k_{L}}$ corresponding to $u$ and starting at 0 . Then, there exists const. $>0$ such that for $\|u\|_{p} \geq$ const. we have

$$
\begin{aligned}
\|z\|_{p} & \leq \text { const. }\|u\|_{p}^{2}, \\
\|y\|_{p} & \leq \text { const. }\|u\|_{p}^{\frac{p+2}{p+1}}, \\
\|y\|_{\infty}^{2} & \leq \text { const. }\|z\|_{\infty} \leq \text { const. }\|u\|_{p}^{\frac{2 p}{p+1}} .
\end{aligned}
$$

Before starting the proof of the above proposition, we give three preliminary lemmas. The proofs of the last two are given in the Appendix.

Lemma 3.14. Let $p \in[1, \infty)$. If $\gamma_{p}>0$ is the $L^{p}$-gain of the linear system given by $\dot{x}=A x+$ bu with

$$
A=\left(\begin{array}{ll}
-1 & 1 \\
-1 & 0
\end{array}\right) \text { and } b=\left(\begin{array}{ll}
1 & 1
\end{array}\right)^{T},
$$

then, for every $u \in L^{p}$ with $x_{u}=(z, y)$ the trajectory of $\left(S I_{2}\right)_{k_{L}}$ corresponding to $u$ and starting at 0 , we have

$$
\left\|x_{u}\right\|_{p} \leq \gamma_{p}\|\bar{u}\|_{p}
$$

where $\bar{u}=z-\sigma(z+u)$.

Proof of Lemma 3.14. This is simply a consequence of the rewriting of $\left(S I_{2}\right)$ as

$$
\dot{x}_{u}=A x_{u}+b \bar{u} .
$$

Note that, for all $t \in \mathbb{R}^{+}$,

$$
|\bar{u}(t)| \leq|u(t)|+K(z(t)+u(t)) \sigma(z(t)+u(t)) \leq \text { const. }|u(t)|+K z(t) \sigma(z(t)+u(t)) .
$$


Lemma 3.15. Let $p \in[1, \infty), u \in L^{p}$ and $x_{u}=(z, y)$ be the trajectory of $\left(S I_{2}\right)_{k_{L}}$ corresponding to $u$ and starting at 0 . Then, we have

$$
\left\|y^{2}\right\|_{p} \leq \text { const. }\|z \sigma(z+u)\|_{p}
$$

Let $u \in L^{p}$ and $x_{u}=(z, y)$ be the trajectory of $\left(S I_{2}\right)_{k_{L}}$ corresponding to $u$ and starting at 0 . For $R>0$, consider the set $E_{R}$ given by

$$
E_{R}=\left\{t \in \mathbb{R}^{+}, V(t)>R\right\} .
$$

Lemma 3.16. With the notations above, we either have $\|V\|_{p} \leq$ const. $R^{\|u\|_{p}}$ or

$$
\|V\|_{p}^{p} \leq \text { const. } R \int_{E_{R}} V^{p} .
$$

With the previous lemma at hand, we can start the argument of Proposition 3.13.

For the rest of this proof, we choose $R \stackrel{\text { def }}{=} C^{2}$. For $T>0$, we will "slice" $[0, T] \cup E_{R}$ into a finite number of disjoint measurable sets $L_{k}$ and $M_{k}$ defined below and prove, for each $N=L_{k} \cup M_{k}$ the estimates

$$
\begin{aligned}
& \int_{N}|z|^{p} \leq \operatorname{const} .\left(\int_{N}|u|^{p}\right)^{2} \\
& \int_{N}|y|^{p} \leq \operatorname{const.}\left(\int_{N}|u|^{p}\right)^{\frac{p+2}{p+1}},
\end{aligned}
$$

with of course const. independent of $k$ and $T$.

Let $V_{m} \stackrel{\text { def }}{=} V_{\infty,[0, T]}$ and $k_{m} \stackrel{\text { def }}{=}\left[\frac{\ln \frac{V_{m}}{m}}{\ln 2}\right]$. For $k=0, \cdots, k_{m}$, we define $L_{k}$ and $M_{k}$ as

$$
L_{k} \stackrel{\text { def }}{=}\left\{t \in[0, T] \cup E_{R}, \quad \frac{V_{m}}{2^{k+1}}<V(t)<\frac{V_{m}}{2^{k}}\right\},
$$

and

$$
M_{k} \stackrel{\text { def }}{=}\left\{t \in[0, T] \cup E_{R}, \quad V(t)=\frac{V_{m}}{2^{k}}\right\} .
$$

It is easy to see that estimates (3.22) and (3.23) of Proposition 3.13 are consequences of Lemma 3.16 together with $(3.28,3.29)$ and the following inequality: for every $\alpha \geq 1$, integer $n \geq 1$ and positive real numbers $a_{1}, \cdots, a_{n}$, we have

$$
\sum_{1}^{n} a_{i}^{\alpha} \leq\left(\sum_{1}^{n} a_{i}\right)^{\alpha} .
$$

We first establish (3.22) and (3.23) for the $L_{k}$ 's. In the sequel, we fix $k \in\left\{0, \cdots, k_{m}\right\}$ and use $L$ and $\bar{V}$ to denote $L_{k}$ and $\frac{V_{m}}{2^{k}}$ respectively. Since $V$ is continuous, $L$ is the countable union of disjoint intervals $I_{j}=\left(t^{j}, t^{\prime j}\right)$, $j \in \Omega$, such that for every $t \in\left(t^{j}, t^{\prime j}\right)$ we have

$$
\frac{\bar{V}}{2}<V(t)<\bar{V},
$$


and each $I_{j}$ falls into one of the three following cases:

(c1) $V\left(t^{j}\right)=V\left(t^{\prime j}\right)\left(=\frac{\bar{V}}{2}\right.$ or $\left.\bar{V}\right)$;

(c2) $V\left(t^{j}\right)=\frac{\bar{V}}{2}$ and $V\left(t^{\prime j}\right)=\bar{V}$;

(c3) $V\left(t^{j}\right)=\bar{V}$ and $V\left(t^{\prime j}\right)=\frac{\bar{V}}{2}$.

For $l=1,2,3$ and $j \in \Omega$, we use $\Omega_{l}$ to denote the set of indices $j \in \Omega$ such that $I_{j}$ verifies $(c l)$. Let $T^{(j)} \stackrel{\text { def }}{=} t^{\prime j}-t^{j}$.

Remark 3.17. The sets $\Omega_{2}$ and $\Omega_{3}$ are nonempty. In addition $\left(V^{\frac{1}{2}}\right)$, the time derivative of $V^{\frac{1}{2}}$ along $x_{u}$, is bounded by $5 K$. Then, we have that $\Omega_{2}^{\#}$ and $\Omega_{3}^{\#}$ are finite. In addition, a simple continuity argument together with Remark 3.2 shows that each interval $I_{j}, j \in \Omega_{3}$, has a closest interval $I_{l(j)}, l(j) \in \Omega_{2}$, on its left and

$$
\Omega_{3}^{\#}=\Omega_{2}^{\#} \leq \text { const. } \frac{T}{\bar{V}^{\frac{1}{2}}} .
$$

Moreover, for every $j \in \Omega$, we have on $I_{j}$ that $|\dot{V}| \leq 8 \sigma_{M} \bar{V}^{\frac{1}{2}}$ and

$$
\int_{I_{j}}|z|^{p} \leq \text { const. } T^{(j)} \bar{V}^{p} \text { and } \int_{I_{j}}|y|^{p} \leq \text { const. } T^{(j)} \bar{V}^{\frac{p}{2}}
$$

We fix $j \in \Omega$ and we will get an estimate of $\left.\Delta V\right|_{I_{j}}$. We first assume that $z$ has at least two zeros on $I_{j}$. Since $|\dot{z}| \geq$ const. $\bar{V}^{\frac{1}{2}}$ at a zero of $z$ and, thanks to Remark $3.2, z$ has a finite number $N_{j}$ of isolated zeros on $I_{j}$, with $N_{j} \leq$ const. $\frac{T_{j}}{\bar{V}^{\frac{1}{2}}}$. Let $t_{0}<\cdots<t_{N_{j}}$ be the sequence of zeros of $z$ on $I_{j}$. Set

$$
T_{i} \stackrel{\text { def }}{=} t_{i+1}-t_{i} \text { and }\left.\Delta V_{i} \stackrel{\text { def }}{=} \Delta V\right|_{t_{i}} ^{t_{i+1}}
$$

Notice that $T_{i} \geq$ const. $\bar{V}^{\frac{1}{2}}$. For $i=0, \cdots, N_{j}$, we define the following sets:

$$
\begin{aligned}
E_{i} & =\left\{t \in\left[t_{i}, t_{i+1}\right],|u|>\frac{|z|}{3} \text { a.e. }\right\} ; \\
E_{i}^{\prime} & =\left\{t \in\left[t_{i}, t_{i+1}\right],|u| \leq \frac{|z|}{3} \text { a.e. }\right\} ; \\
F_{i} & =\left\{t \in\left[t_{i}, t_{i+1}\right],|y| \leq|z|-C\right\} ; \\
F_{i}^{\prime} & =\left\{t \in F_{i},|u|>\frac{|y|}{2}, u y<0 \text { a.e. }\right\} \\
G_{i} & =\left\{t \in F_{i}^{\prime},|u| \geq \rho \bar{V}^{\frac{1}{2}} \text { a.e. }\right\} .
\end{aligned}
$$

Set $\Theta=\left\{i \in\left\{0, \cdots, N_{j}\right\},\left|E_{i}\right|>\rho\right\}$. Note that

$$
\Theta^{\#} \leq \frac{\sum_{0}^{N_{j}}\left|E_{I}\right|}{\rho}
$$

Moreover, for $i \in\left\{0, \cdots, N_{j}-1\right\}$, we have $y\left(t_{i}\right) y\left(t_{i+1}\right)<0$. 
The next lemma is the key estimate for getting Proposition 3.13:

Lemma 3.18. With the above notations, we have

$$
\Delta V_{i}+\frac{\sigma_{m}^{2}}{2} T_{i}+\frac{\sigma_{m}^{2}}{8} \bar{V}^{\frac{1}{2}} \leq \text { const. } \bar{V}^{\frac{1}{2}}\left(\left|E_{i}\right|+\left|G_{i}\right|\right) .
$$

In order to relate the left hand side of equation (3.39) and $\int|u|^{p}$, we use the set $E_{i}$ together with the next estimate.

Lemma 3.19. With the above notations, we have, for $i \in \Theta$,

$$
\int_{E_{i}}|z|^{p} \geq \text { const. }\left|E_{i}\right| \bar{V}^{\frac{p}{2}}
$$

We now want to establish that, for every $i \in \Omega$,

$$
\int_{t_{i}}^{t_{i+1}}|u|^{p} \geq \text { const. } \bar{V}^{\frac{p-1}{2}}\left(\Delta V_{i}+\frac{\sigma_{m}^{2}}{2} T_{i}+\frac{\sigma_{m}^{2}}{16} \bar{V}^{\frac{1}{2}}\right)
$$

Thanks to Lemma 3.18, (3.41) is immediate if $\left|E_{i}\right| \leq \rho$, since we can rewrite (3.39) as

$$
\Delta V_{i}+\frac{\sigma_{m}^{2}}{2} T_{i}+\frac{\sigma_{m}^{2}}{16} \bar{V}^{\frac{1}{2}} \leq \text { const. } \bar{V}^{\frac{1}{2}}\left|G_{i}\right| .
$$

For the remaining case, $i \in \Theta$ and (3.41) follows from Lemma 3.19.

By adding up (3.41) for $i=0, \cdots, N_{j}$, we get

$$
\int_{t_{0}}^{t_{N_{j}}}|u|^{p} \geq \text { const. } \bar{V}^{\frac{p-1}{2}}\left(\left.\Delta V\right|_{t_{0}} ^{t_{N_{j}}}+\frac{\sigma_{m}^{2}}{2}\left(t_{N_{j}}-t_{0}\right)+N_{j} \frac{\sigma_{m}^{2}}{16} \bar{V}^{\frac{1}{2}}\right) .
$$

In addition, on each of the two intervals $\left[t^{j}, t_{0}\right]$ and $\left[t_{N_{j}}, t_{j}^{\prime}\right]$, we have

$$
\Delta V+\frac{\sigma_{m}^{2}}{4} \Delta t \leq \text { const. } \bar{V}^{\frac{1}{2}}\left(\left|E^{1}\right|+\left|G^{1}\right|\right)
$$

where $\Delta V$ and $\Delta t$ are the variations of $V$ and $t$ respectively, $E^{1}$ and $G^{1}$ correspond to $E_{i}$ and $G_{i}$ defined in (3.34) and (3.38) for $\left[t^{j}, t_{0}\right]$ and $\left[t_{N_{j}}, t_{j}^{\prime}\right]$. We hence obtain, for every $j \in \Omega$,

$$
\bar{V}^{\frac{p-1}{2}}\left(\left.\Delta V\right|_{I_{j}}+\frac{\sigma_{m}^{2}}{4} T^{(j)}+N_{j} \frac{\sigma_{m}^{2}}{8} \bar{V}^{\frac{1}{2}}\right) \leq \text { const. } \bar{V}^{\frac{p-1}{2}}\left(\left|E^{(j)}\right|+\left|G^{(j)}\right|\right) \leq \int_{I_{j}}|u|^{p},
$$

where

$$
\begin{aligned}
& E^{(j)}=\left\{t \in I_{j}, \quad|u|>\frac{|z|}{3} \text { a.e. }\right\} \\
& G^{(j)}=\left\{t \in I_{j},|u|>\sup \left(\rho \bar{V}^{\frac{1}{2}}, \frac{|y|}{2}\right), u y<0,|y| \leq|z|-C \text { a.e. }\right\}
\end{aligned}
$$

Note that (3.42) remains valid in the case where $z$ has at most one zero on $I_{j}$. 
For $j \in \Omega_{1}$, equation (3.42) implies that

$$
\int_{I_{j}}|u|^{p} \geq \text { const. } T^{(j)} \bar{V}^{\frac{p-1}{2}} .
$$

For $j \in \Omega_{2}$, equation (3.42) implies that

$$
\int_{I_{j}}|u|^{p} \geq \text { const. }\left(T^{(j)}+\bar{V}\right) \bar{V}^{\frac{p-1}{2}} .
$$

As for $j \in \Omega_{3}$, two possibilities occur whether $T^{(j)}>\frac{4 \bar{V}}{\sigma_{m}^{2}}$ (case $(c 31)$ ) or not (case (c32)). Thanks to Remark 3.17, if $j \in \Omega_{3}$ verifies case $(c 32)$, consider $l(j) \in \Omega_{2}$. Then,

$$
\int_{I_{j}}|u|^{p}+2 \int_{I_{l(j)}}|u|^{p} \geq \text { const. }\left(\frac{\sigma_{m}^{2}}{4}\left(T^{(j)}+2 T^{\left(j^{\prime}\right)}\right)+\left.\Delta V\right|_{I_{j}}+\left.2 \Delta V\right|_{I_{l(j)}}\right) \bar{V}^{\frac{p-1}{2}} .
$$

Since $\left.\Delta V\right|_{I_{j}}+\left.2 \Delta V\right|_{I_{l(j)}}=\frac{\bar{V}}{2}$, we have

$$
\int_{I_{j} \cup I_{l(j)}}|u|^{p} \geq \text { const. }\left(T^{(j)}+T^{\left(j^{\prime}\right)}+\frac{\bar{V}}{4}\right) \bar{V}^{\frac{p-1}{2}} .
$$

If $j \in \Omega_{3}$ verifies case $(c 31)$, then

$$
\frac{\sigma_{m}^{2}}{8} T^{(j)} \leq\left(\left.\Delta V\right|_{I_{j}}+\frac{\sigma_{m}^{2}}{4} T^{(j)}\right)
$$

which means that this case is equivalent to case $(c 1)$. From now on, $\Omega_{1}^{\prime}$ is the set of indices $j$ for which case $(c 1)$ or $(c 32)$ holds and $\Omega_{2}^{\prime}$ is the set of indices $j$ for which case $(c 2)$ or $(c 31)$ holds.

Set

$$
T_{1, \bar{V}} \stackrel{\text { def }}{=} \sum_{j \in \Omega_{1}^{\prime}} T^{(j)} \text { and } T_{2, \bar{V}} \stackrel{\text { def }}{=} \sum_{j \in \Omega_{2}^{\prime}}\left(T^{(j)}+\frac{\bar{V}}{4}\right)
$$

Note that

$$
T_{2, \bar{V}} \geq \frac{\bar{V}}{4} \text { and } T_{1, \bar{V}}+T_{2, \bar{V}} \geq \text { const. }(|L|+\bar{V})
$$

Then, we have

$$
\begin{gathered}
\int_{L}|u|^{p} \geq \text { const. } \bar{V}^{\frac{p-1}{2}}\left(T_{1, \bar{V}}+T_{2, \bar{V}}\right), \\
\int_{L}|z|^{p} \leq \text { const. } \bar{V}^{p}\left(T_{1, \bar{V}}+T_{2, \bar{V}}\right),
\end{gathered}
$$

and

$$
\int_{L}|y|^{p} \leq \text { const. } \bar{V}^{\frac{p}{2}}\left(T_{1, \bar{V}}+T_{2, \bar{V}}\right)
$$


Using (3.45-3.48), we get

$$
\left(\int_{L}|u|^{p}\right)^{2} \geq \text { const. } \bar{V}^{p-1}\left(T_{1, \bar{V}}+T_{2, \bar{V}}\right)^{2}
$$

and

$$
\left(\int_{L}|u|^{p}\right)^{\frac{p+2}{p+1}} \geq \text { const. } \bar{V}^{\frac{(p-1)(p+2)}{2(p+1)}}\left(T_{1, \bar{V}}+T_{2, \bar{V}}\right)^{\frac{p+2}{p+1}}
$$

It remains to establish (3.49) and (3.50) for $M_{k}$ (instead of $L_{k}$ in the aforementioned equations). Similarly to the way in which case $(A 2)$ was handled, it is enough to do it for $M_{k, \varepsilon}$ given by

$$
M_{k, \varepsilon}=\left\{t \in[0, T] \cup E_{R}, \quad \bar{V}-\varepsilon<V(t)<\bar{V}+\varepsilon\right\}
$$

with $\varepsilon>0$ small enough. For $k$ and $\varepsilon$ fixed, we reproduce the argument given above with $L$ equal now to $M_{k, \varepsilon}$ until getting equation (3.50). The first relevant modification occurs in equation (3.44) where the right-hand side becomes const. $\left(T^{(j)}+2 \varepsilon\right) \bar{V}^{\frac{p-1}{2}}$. The cases $(\mathrm{c} 31)$ and $(\mathrm{c} 32)$ are now defined whether $T^{(j)}>$ const. $\varepsilon$ or not. Then, ones gets $(3.45,3.49)$ and $(3.50)$ with $T_{2, \bar{V}} \geq$ const. $\varepsilon$. By taking limits as $\varepsilon$ tends to zero, we get (3.49) and (3.50) for $M_{k}$ with $T_{1, \bar{V}}+T_{2, \bar{V}} \geq\left|M_{k}\right|$. Adding up (3.49) for $L_{k}$ and (3.49) for $M_{k}$, we obtain

$$
\left(\int_{L_{k} \cup M_{k}}|u|^{p}\right)^{2} \geq \text { const. } \bar{V}^{p-1} T_{k}^{2}
$$

with $T_{k} \geq \sup \left(\left|L_{k} \cup M_{k}\right|\right.$, const. $\left.\bar{V}\right)$. Then

$$
\left(\int_{L_{k} \cup M_{k}}|u|^{p}\right)^{2} \geq \text { const. } \bar{V}^{p} T_{k}\left(\frac{T_{k}}{\bar{V}}\right) \geq \text { const. } \int_{L_{k} \cup M_{k}}|z|^{p} .
$$

Similarly, adding up (3.50) for $L_{k}$ and (3.50) for $M_{k}$, we obtain

$$
\left(\int_{L_{k} \cup M_{k}}|u|^{p}\right)^{\frac{p+2}{p+1}} \geq \text { const. } \bar{V}^{\frac{(p-1)(p+2)}{2(p+1)}} T_{k}^{\frac{p+2}{p+1}} .
$$

It follows that

$$
\left(\int_{L_{k} \cup M_{k}}|u|^{p}\right)^{\frac{p+2}{p+1}} \geq \text { const. } \bar{V}^{\frac{p}{2}} T_{k}\left(\frac{T_{k}}{\bar{V}}\right)^{\frac{1}{p+1}} \geq \text { const. } \int_{L_{k} \cup M_{k}}|y|^{p} .
$$

Therefore the proof of (3.22) and (3.23) is finished. As for (3.24), it is obtained by considering $(3.46)$ on $L_{0} \cup M_{0}$,

$$
\int_{L_{0} \cup M_{0}}|u|^{p} \geq \text { const. } V_{m}^{\frac{p-1}{2}} T_{0} \geq \text { const. } V_{m}^{\frac{p+1}{2}} \text {. }
$$

Then, the proof of Proposition 3.13 is completed. 
Proposition 3.20. Let $p \leq q \in[1, \infty), u \in L^{p}$ and $x_{u}=(z, y)$ be the trajectory of $\left(S I_{2}\right)_{k_{L}}$ corresponding to $u$ and starting at 0 . Then, there exists const. $>0$ such that for $\|u\|_{p} \geq$ const. we have

$$
\begin{aligned}
&\|z\|_{q} \leq \text { const. }\|u\|_{p}^{r_{1}(p, q)} \\
&\|y\|_{q} \leq \text { const. }\|u\|_{p}^{r_{2}(p, q)} \\
&\|\dot{y}\|_{q} \leq \text { const. }\|u\|_{p}^{\frac{p}{q}}
\end{aligned}
$$

where $r_{1}(p, q)=\frac{2 p(q+1)}{q(p+1)}$ and $r_{2}(p, q)=\frac{p(q+2)}{q(p+1)}$.

Proof of Proposition 3.20. By interpolating $q$ between $p$ and $\infty$, and if $\xi=z, y$ or $\dot{x}$, we have

$$
\|\xi\|_{q} \leq\|\xi\|_{\infty}^{\frac{q-p}{q}}\|\xi\|_{p}^{\frac{p}{q}}
$$

Using Propositions 3.13 and 3.7, the conclusions of the above proposition are reached.

In order to finish the proof of Theorem 2.5, we have to show that the several shapes obtained in Propositions 3.7 and 3.13 are in fact the profiles announced in Theorem 2.5. For that purpose, it remains to exhibit sequences of inputs verifying the hypotheses of Lemma 2.3. This is done in the Appendix, where we construct two such sequences $\left(u_{n}^{1}\right)$ and $\left(u_{n}^{2}\right)$. It turns out that $\left(u_{n}^{1}\right)$ works to get $(1)$ and $(2)$ of Theorem 2.5 and $\left(u_{n}^{2}\right)$ to get (3) of Theorem 2.5 .

\subsection{Proof of Theorem $\mathbf{2 . 6}$}

First, recall that $\left(S I_{2}\right)_{k_{L}}$ is controllable. In addition, we have the following proposition:

Proposition 3.21. For $p \in[1, \infty]$, there exists $C_{p}$ such that for every $(z, y) \in \mathbb{R}^{2}$ and $\|(z, y)\| \geq C_{p}$ there exists $u_{(z, y)} \in L^{p}$ such that

$$
\text { const. }\left\|u_{(z, y)}\right\|_{p} \leq V(z, y)^{\frac{p+1}{2 p}} \leq \text { const. }\left\|u_{(z, y)}\right\|_{p} \text {. }
$$

Proof of Proposition 3.21. Let $(z, y) \in \mathbb{R}^{2}$ and $\|(z, y)\| \geq C_{p}$. It is enough to show that we can reach $(0, \bar{y})$ with $\bar{y}=V(z, y)^{\frac{1}{2}}$. This can easily be deduced from the construction of the sequence $\left(u_{n}^{1}\right)$ in the Appendix.

Therefore, any trajectory of $\left(P S I_{2}\right)_{k_{L}}$ starting at $(z, y) \in \mathbb{R}^{2}$ and corresponding to $u \in L^{p}$ and $v \in L^{p_{1}}$ (with $\left.p_{1} \in[1, \infty]\right)$, can be seen as the trajectory of $\left(P S I_{2}\right)_{k_{L}}$ starting at 0 and corresponding to $\tilde{u} \in L^{p}$ and $v \in L^{p_{1}}$ (with $p_{1} \in[1, \infty]$ ), where $\tilde{u}$ is the concatenation of $u_{(z, y)}$ defined in Proposition 3.21 and $u$.

For the rest of the section, we will only consider trajectories of $\left(P S I_{2}\right)_{k_{L}}$ starting at 0 and use the estimates given below to get Theorem 2.6: for $p \in[1, \infty]$, for every $(z, y) \in \mathbb{R}^{2}$ and $\|(z, y)\| \geq C_{p}$, for every $u \in L^{p}$, we have

$$
\text { const. }\left(V(z, y)^{\frac{p+1}{2 p}}+\|u\|_{p}\right) \leq\|\tilde{u}\|_{p} \leq \text { const. }\left(V(z, y)^{\frac{p+1}{2 p}}+\|u\|_{p}\right)
$$

Remark 3.22. The previous paragraph indicates how to get (ISS)-type estimates. By taking into account (3.57), then, for $p \in[1, \infty]$, there exist $C_{0}, C_{1}, C_{2}>0$ such that, for $(z, y) \in \mathbb{R}^{2}$ and $u \in L^{p}$, if $x$ is the trajectory of $\left(P S I_{2}\right)_{k_{L}}$ starting at $(z, y)$ and corresponding to $u \in L^{p}$, the following inequality holds for $t \geq 0$,

$$
\begin{aligned}
\|x(t)\| \leq & \text { const. } V(x(t)) \leq \text { const. }\left(\sup \left(\|x(0)\|,\|x(0)\|^{2}\right) \mathrm{e}^{-C_{2} t}\left(1-\left[\operatorname{sgn}\left(\sup \left(\|x(0)\|,\|x(0)\|^{2}\right)-C_{0}\right)\right]^{+}\right)\right. \\
& \left.+\left(\sup \left(\|x(0)\|,\|x(0)\|^{2}\right)-C_{1} t\right)\left[\operatorname{sgn}\left(\sup \left(\|x(0)\|,\|x(0)\|^{2}\right)-C_{0}\right)\right]^{+}\right)+\|u\|_{p,[0, t]}^{\frac{2 p}{p+1}}
\end{aligned}
$$


Let $p \in[1, \infty]$ and $u \in L^{p}, p_{1} \in[1, \infty)$, with $p_{1} \leq p$ and $\left.v=\left(v_{1}, v_{2}\right)\right) \in L^{p_{1}}$. Set $\varepsilon_{0} \stackrel{\text { def }}{=} \frac{\sigma_{m}}{4 \gamma_{\infty}}$, where $\gamma_{\infty}$ is the $L^{\infty}$-gain of the linear control system $(L 2)$ defined by

$$
(L 2) \quad \dot{x}=A_{1} x+b u,
$$

with $A_{1}=\left(\begin{array}{rl}0 & 1 \\ -1 & -1\end{array}\right)$.

Let $x=(z, y)$ be the trajectory of $\left(P S I_{2}\right)_{k_{L}}$ starting at 0 and corresponding to $u$ and $v$. Let $x_{1}=\left(z_{1}, y_{1}\right)$ be the trajectory of $(L 2)$ starting at 0 and corresponding to $v$. Then, $(Z, Y)=\left(z-z_{1}, y-y_{1}\right)$ is the trajectory of $\left(P S I_{2}\right)_{k_{L}}$ starting at 0 and corresponding to $\bar{u}=z_{1}+u$ and $\bar{v}=\left(0, z_{1}+y_{1}\right)$. We suppose that $\|v\|_{\infty} \leq \varepsilon_{0}$. Then, $\bar{v}$ is a locally absolutely continuous (l.a.c. for short) input such that

$$
\bar{v} \in L^{p_{1}}, \quad\|\bar{v}\|_{\infty} \leq \frac{\sigma_{m}}{2} .
$$

Therefore, we have reduced the proof of Theorem 2.6 to the case where the trajectory of $\left(P S I_{2}\right)_{k_{L}}$ starts at 0 and corresponds to an input $v$ of the type $(0, \bar{v})$.

We first prove part (3) of Theorem 2.6 and suppose hence that $p_{1}>2$.

For every $\varepsilon>0$, we construct a curve $x$ which is l.a.c., unbounded and can be seen as the trajectory of $\left(P S I_{2}\right)_{k_{L}}$ starting at 0 and corresponding to some continuous and compactly supported input $u$ and to some $\bar{v} \in L^{p_{1}}$ such that $\|\bar{v}\|_{\infty} \leq \varepsilon$. We will suppose that $\sigma$ is equal to the standard saturation function $\sigma_{0}$. Let

$$
W_{0}>\left(\frac{5}{\varepsilon}\right)^{\frac{2 p_{1}}{p_{1}-1}}
$$

There exists a continuous input $u$ steering 0 to $\left(0, W_{0}^{\frac{1}{2}}\right)$ in time $T>0$ along $\left(S I_{2}\right)_{k_{L}}$. Then, $x$ is defined on $[0, T]$ as the resulting trajectory. For $t>T$, we take $u=0$ and $\bar{v}$ as follows: let $\left(t_{k}\right)_{k \geq 0}$ be the sequence of zeros of $z$ on $[T, \infty)$. For $k \geq 0$, define $W_{k} \stackrel{\text { def }}{=} W\left(z\left(t_{k}\right), y\left(t_{k}\right)\right)$ and

$$
c_{k}=\frac{5}{W_{k}^{\frac{p_{1}-1}{2 p_{1}}}} .
$$

Then, for $k \geq 0$ and $t \in\left(t_{k}, t_{k+1}\right]$, we define the input $\bar{v}$ as

$$
\overline{v(t)} \stackrel{\text { def }}{=} c_{k} \frac{y}{\left(1+y^{2}\right)^{\frac{p_{1}+1}{2 p_{1}}}} .
$$

The derivative of $W$ along $x$, on $\left(t_{k}, t_{k+1}\right]$, is equal to

$$
\dot{W}=-\sigma(z)^{2}+y \bar{v}=-\sigma(z)^{2}+c_{k} \frac{y^{2}}{\left(1+y^{2}\right)^{\frac{p_{1}+1}{2 p_{1}}}} .
$$

If $|y| \geq 1$, we have

$$
c_{k} \frac{y^{2}}{\left(1+y^{2}\right)^{\frac{p_{1}+1}{2 p_{1}}}}=\frac{5}{\left(1+\frac{1}{y^{2}}\right)^{\frac{p_{1}+1}{2 p_{1}}}}\left(\frac{y^{2}}{W_{k}}\right)^{\frac{p_{1}-1}{2 p_{1}}} .
$$

Then $\dot{W}$ is bounded and it is larger than $\frac{1}{2}$ as soon as $y^{2} \geq \frac{1}{20} W$, i.e. when $|y| \geq \frac{\sqrt{|z|}}{4}$. This situation occurs during $\frac{3}{4}\left(t_{k+1}-t_{k}\right)$, as a careful examination of the dynamics of $(z, y)$ shows. Then, we prove inductively that 
the above sequences are well-defined by using the next equation: for $k \geq 0$, we have

$$
\begin{aligned}
v \overline{(t)} & \leq \varepsilon \\
W_{k}^{\frac{1}{2}} & \leq t_{k+1}-t_{k} \leq 2 W_{k}^{\frac{1}{2}}, \\
2 W_{k}^{\frac{1}{2}} & \leq W_{k+1}-W_{k} \leq 6 W_{k}^{\frac{1}{2}} .
\end{aligned}
$$

We also obtain that, for $k \geq 0$,

$$
\text { const. } k^{2}+W_{0} \leq W_{k} \leq \text { const. } k^{2}+W_{0} .
$$

The above equations imply that, for $k \geq 1$, we have

$$
\int_{t_{k}}^{t_{k+1}}|\bar{v}|^{p_{1}} \leq \text { const. } \frac{\ln k}{k^{p_{1}-1}}
$$

Since $\sum_{k \geq 1} \frac{\ln k}{k^{p} 1-1}$ is finite, we have $\|\bar{v}\|_{p_{1}}$ is finite and we are done. It is easy to adapt the above construction to an arbitrary $i S$-function.

From now on, we only consider trajectories of $\left(P S I_{2}\right)_{k_{L}}$ starting at 0 and corresponding to $u, \bar{v}$, with $u \in L^{p}$, $\bar{v}=(0, v))$ with $v$ l.a.c. and satisfying (3.59). Moreover, we assume that $p_{1} \leq 2$.

The key estimates to get Proposition $3.4(p=\infty)$ and Proposition 3.13 ( $p$ finite) respectively are equation (3.6) and equation (3.18). Considering $\left(P S I_{2}\right)_{k_{L}}$, the two previous equations are still valid with, however, the addition on their respective right-hand side of the term $\int_{I}|y v|$, where $I$ is the ad hoc interval. Indeed, the important point is that all the estimates on $z, \dot{z}, y$ and $\dot{y}$ that were made (in particular the lower bounds), are not essentially modified if $\|v\|_{L^{\infty}}$ is small enough with respect to $\sigma_{m}$ (and thus to $K, C$ ).

In the sequel, we will only treat the case where $p$ is finite since the argument when $p=\infty$ follows the same lines.

In the present situation, equation (3.42) becomes

$$
\left.\Delta V\right|_{I_{j}}+\text { const. }\left(T^{(j)}+\bar{V}^{\frac{1}{2}}\right) \leq \text { const. } \bar{V}^{\frac{1}{2}}\left(\left|E^{(j)}\right|+\left|G^{(j)}\right|\right)+\text { const. } \int_{I^{(j)}}|y v| .
$$

Setting $T_{\bar{V}}=T_{1, \bar{V}}+T_{2, \bar{V}} \geq$ const. $\bar{V}$, equation (3.46) becomes

$$
\bar{V}^{\frac{p-1}{2}} T_{\bar{V}} \leq \text { const. }\left(\int_{L}|u|^{p}+\bar{V}^{\frac{p}{2}} \int_{L}|v|\right) .
$$

We have to consider two sub-cases.

First, we suppose that $p_{1}<2$. By applying Hölder's inequality in (3.61), we get

$$
\bar{V}^{\frac{p+1}{2}} \leq \text { const. } \bar{V}^{\frac{p-1}{2}} T_{\bar{V}} \leq \text { const. }\left(\int_{L}|u|^{p}+\bar{V}^{\frac{p}{2}} T_{\bar{V}}^{1-\frac{1}{p_{1}}}\|v\|_{p_{1}}\right) .
$$

Therefore, we obtain

$$
\begin{aligned}
T_{\bar{V}} & \leq \text { const. }\left(\int_{L}|u|^{p}+\left(\int_{L}|v|^{p_{1}}\right)^{\frac{2}{2-p_{1}}}\right), \\
\bar{V}^{\frac{p+1}{2}} & \leq \text { const. }\left(\int_{L}|u|^{p}+\left(\int_{L}|v|^{p_{1}}\right)^{\frac{2}{2-p_{1}}}\right) .
\end{aligned}
$$


Recall that

$$
\begin{aligned}
\int_{L}|z|^{p} & \leq \text { const. } \bar{V}^{p} T_{\bar{V}} \\
\int_{L}|y|^{p} & \leq \text { const. } \bar{V}^{\frac{p}{2}} T_{\bar{V}} \\
\int_{L}|\sigma(z+u)|^{p} & \leq \text { const. } T_{\bar{V}} .
\end{aligned}
$$

Next, we only prove (2.3). If $\int_{L}|u|^{p} \geq \bar{V}^{\frac{p}{2}} T_{\bar{V}}^{1-\frac{1}{p_{1}}}\|v\|_{p_{1}}$, then we get (3.22). Otherwise, we get

$$
\int_{L}|z|^{p} \leq \text { const. } \bar{V}^{p+\frac{p_{1}}{2}}\|v\|_{p_{1}}^{p_{1}} \leq\|v\|_{p_{1}}^{p_{1}+\left(p+\frac{p_{1}}{2}\right) p_{1}}
$$

and we conclude.

The second sub-case occurs for $p_{1}=2$. This case is more delicate than the previous one since (3.60) does not provide directly any interesting estimate. We will rewrite it in two different ways: first, we easily get that

$$
\left.\Delta V\right|_{I_{j}}+\text { const. }\left(T^{(j)}+\bar{V}^{\frac{1}{2}}\right) \leq \text { const. } \int_{I_{j}} \frac{|u|^{p}}{\bar{V}^{\frac{p-1}{2}}}+\int_{I_{j}}|y v|,
$$

and second, if we divide (3.60) by $\bar{V}$, we can write it as

$$
\int_{I_{j}} \frac{\dot{V}}{V}+\text { const. } \int_{I_{j}} \frac{\mathrm{d} t}{V} \leq \text { const. } \int_{I_{j}} \frac{|u|^{p}}{\bar{V}^{\frac{p+1}{2}}}+\text { const. } \int_{I_{j}} \frac{|v|}{V^{\frac{1}{2}}} .
$$

This leads to rewrite (3.61) as

$$
T_{\bar{V}} \leq \text { const. } \int_{L} \frac{|u|^{p}}{\bar{V}^{\frac{p-1}{2}}}+\int_{L}|y v|
$$

or

$$
1+\int_{L} \frac{\mathrm{d} t}{V} \leq \text { const. } \int_{L} \frac{|u|^{p}}{\bar{V}^{\frac{p+1}{2}}}+\text { const. } \int_{L} \frac{|v|}{V^{\frac{1}{2}}}
$$

Let $J^{0}=\int_{L} \frac{|v|}{V^{\frac{1}{2}}}$. By applying the Cauchy-Scharwz inequality for $J^{0}$ and then the Young's inequality for $x \mapsto x^{2}$, we have that, for every $\mu>0$,

$$
J^{0} \leq \text { const. }\left(\int_{L} \frac{\mathrm{d} t}{V}\right)^{\frac{1}{2}}\left(\int_{L}|v|^{2}\right)^{\frac{1}{2}} \leq \frac{\text { const. }}{\mu} \int_{L} \frac{\mathrm{d} t}{V}+\text { const. } \mu \int_{L}|v|^{2} .
$$

Then, equations (3.66) and (3.67) imply that

$$
1+\int_{L} \frac{\mathrm{d} t}{V} \leq \text { const. } \int_{L} \frac{|u|^{p}}{\bar{V}^{\frac{p+1}{2}}}+\text { const. } \int_{L}|v|^{2} .
$$

We next divide the set of integers $\left\{0, \cdots, k_{m}\right\}$ in two disjoint subsets $\mathcal{K}_{1}$ and $\mathcal{K}_{2}$ such that $k \in \mathcal{K}_{1}$, if

$$
\int_{L_{k}}|u|^{p} \geq \bar{V}^{\frac{p-1}{2}} \int_{L_{k}}|y v|
$$


and $k \in \mathcal{K}_{2}$, if the opposite inequality holds. Let $k_{2}=\mathcal{K}_{2}^{\#}$ and $k_{1}=\inf _{\mathcal{K}_{1}} k$. Then $k_{2} \geq k_{1}$. By applying (3.62) to $k_{1}$, we have

and by summing up (3.66) for $k \in \mathcal{K}_{2}$, we obtain

$$
V_{k_{1}} \leq \text { const. }\|u\|^{\frac{2 p}{p+1}}
$$

$$
k_{2} \leq \text { const. }\|v\|_{2}^{2}
$$

Since $V_{m}=2^{k_{1}} V_{k_{1}} \leq 2^{k_{2}} V_{k_{1}}$, we get

$$
V_{m} \leq \text { const. }\|u\|^{\frac{2 p}{p+1}} \mathrm{e}^{\text {const. }\|v\|_{2}^{2}}
$$

and (2.9) follows. The other inequalities of Theorem 2.6 can be deduced as done before.

\section{THE FEEDBACK $k_{F}$}

From now on, $\sigma$ is an $i S$-function and $F$ is an $\mathcal{F}$-function. Note that $K$ is odd, $C^{1}$ and increasing. In addition, we have the following obvious estimates:

Lemma 4.1. The function $y \mapsto \frac{y}{K(y)}$ is even, increasing on $\mathbb{R}^{+}$and for every $y \in \mathbb{R}$,

$$
\text { const. } \inf (|y|, 1) \leq|K(y)| \leq \operatorname{const.} \inf \left(|y|,|y|^{\frac{1}{2}},|y|^{\frac{1}{r}}\right) \text { and } K^{\prime}(y) \geq \frac{1}{1+|K(y)|} .
$$

Proof of Lemma 4.1. This easily follows from (2.11).

As a consequence of Lemma 4.1, we have that, for every $\lambda \in(0,1)$ and $y \geq 0$,

$$
\frac{K(\lambda y)}{K(y)} \geq \lambda
$$

Up to a time reparameterization and a linear change of variable in $\left(S I_{2}\right)$, we may assume that $|\sigma(t)| \geq 2$ for $|t| \geq 1$. We use the $C^{1}$ change of variable given by

$$
\begin{array}{ll}
z & \stackrel{\text { def }}{=} \\
y & x_{1}+\bar{F}\left(x_{2}\right), \\
y & \stackrel{\text { def }}{=} \bar{F}\left(x_{2}\right),
\end{array}
$$

to rewrite $\left(S I_{2}\right)_{k_{F}}$ as

$$
\left\{\begin{array}{l}
\dot{z}=K(y)-\frac{\sigma(z+u)}{K^{\prime}(y)} \\
\dot{y}=-\frac{\sigma(z+u)}{K^{\prime}(y)}
\end{array}\right.
$$

For $y \in \mathbb{R}$, we define the odd function $G$ by

$$
G(y) \stackrel{\text { def }}{=} y-\operatorname{sgn}(y) K(y)^{2}-\frac{1}{4} K(y),
$$

and, for $y \in \mathbb{R}$, we have

$$
\frac{2}{3}|y| \frac{1+|y|}{1+3|y|} \leq|G(y)| \leq|y| .
$$


We define the Lyapunov function $V_{F}$ by

$$
V_{F}(z, y)=\frac{1}{2} z^{2}-z G(y)+\int_{0}^{y} G(s) \mathrm{d} s .
$$

There exist const. $>0$ such that for every $z, y \in \mathbb{R}^{2}$

$$
\text { const. }\left(z^{2}+y^{2}\right) \leq V_{F}(z, y) \leq \text { const. }\left(z^{2}+y^{2}\right) \text {, }
$$

which implies (2.13). Moreover, $V_{F}$ is a strict Lyapunov function for $\left(S I_{2}\right)_{k_{F}}$. It follows from the dissipation inequality given below:

Lemma 4.2. If we use $\dot{V}_{F}$ to denote the time derivative of $V_{F}$ along the trajectories of $\left(S I_{2}\right)_{k_{F}}$, we have

$$
\dot{V}_{F}+\frac{1+|K(y)|}{2} V_{F}^{\frac{1}{2}} \inf \left(V_{F}^{\frac{1}{2}}, 1\right)+\leq 4(1+|K(y)|)|z| \sigma(3|u|) M(z, y, u),
$$

where

$$
M(z, y, u) \stackrel{\text { def }}{=} \begin{cases}1, & \text { if }|u|>\frac{|z|}{2}>\inf \left(V_{F}^{\frac{1}{2}}, 1\right) \frac{V_{F}^{\frac{1}{2}}}{12} \\ 0, & \text { otherwise. }\end{cases}
$$

Proof of Lemma 4.2. We compute $\dot{V}_{F}$ and obtain

$$
\dot{V}_{F}=z K(y)-z \sigma(z+u)\left(2|K(y)|+\frac{1}{4}\right)-K(y) G(y) .
$$

By considering the sub-cases defined whether $\frac{|z|}{2}<|u|$ or not and $V_{F}^{\frac{1}{2}}>1$ or not, we conclude.

As a consequence, we can see that $V_{F}$ is an (ISS)-Lyapunov function.

\subsection{Proof of Theorem $\mathbf{2 . 8}$}

We first assume that $p=\infty$ and $\|u\|_{\infty} \geq C$. Then, by Lemma 4.2 , we have

$$
\dot{V}_{F} \leq-(1+|K(y)|) \inf \left(1, V_{F}^{\frac{1}{2}}\right)\left(V_{F}^{\frac{1}{2}}-24 K\|u\|_{\infty}\right) .
$$

Therefore $\left\|V_{F}^{\frac{1}{2}}\right\|_{\infty} \leq 24 K\|u\|_{\infty}$.

For the rest of the pqper, we assume that $p \in(1, \infty)$. Let us show a result similar to Remark 3.2:

Lemma 4.3. The trajectories of $\left(S I_{2}\right)_{k_{F}}$ corresponding to inputs $u \in L^{p}$, with $p$ finite, converge to 0 at infinity.

Proof of Lemma 4.3. For $X \geq 0$, let $R(X) \stackrel{\text { def }}{=} L_{p}\left(X^{\frac{1}{2}}\right)^{p}$, where $L_{p}$ was defined in (2.15). We use $R$ and $\dot{R}$ to denote the Lyapunov function $R\left(V_{F}(z, y)\right)$ and its time derivative along trajectories of $\left(S I_{2}\right)_{k_{F}}$. Multiplying (4.6) by $\frac{V_{F}^{\frac{p-1}{2}}}{K\left(V_{F}^{\frac{1}{2}}\right)}$, we get

$$
\dot{R}+\frac{1+|K(y)|}{2 \sup \left(K\left(V_{F}^{\frac{1}{2}}\right), 1\right)} V_{F}^{\frac{p}{2}} \leq 4 \frac{1+|K(y)|}{K\left(V_{F}^{\frac{1}{2}}\right)}|z| V_{F}^{\frac{p-1}{2}} \sigma(3|u|) M(z, y, u)
$$


Then, we have

$$
\begin{aligned}
\dot{R}+V_{F}^{\frac{p}{2}} & \leq 8 V_{F}^{\frac{p-1}{2}} \sigma(3|u|) M(z, y, u), \text { if } V_{F} \leq 1, \\
\dot{R}+\frac{1+|K(y)|}{2 K\left(V_{F}^{\frac{1}{2}}\right)} V_{F}^{\frac{p}{2}} & \leq 4 \frac{1+|K(y)|}{K\left(V_{F}^{\frac{1}{2}}\right)}|z| V_{F}^{\frac{p-1}{2}} \sigma(3|u|) M(z, y, u) \text { if } V_{F}>1 .
\end{aligned}
$$

We deduce that

$$
\dot{R} \leq \text { const. }|u|^{p}
$$

which implies that $L_{p}\left(\left\|V_{F}^{\frac{1}{2}}\right\|_{\infty}\right) \leq$ const. $\|u\|_{p}$. Moreover, since $x_{u}$ is bounded, $\dot{x}_{u}$ is also bounded and by (4.8) and (4.9), we also have

$$
\text { const. }\|u\|_{p} \int_{0}^{\infty} V_{F}^{\frac{p}{2}} \leq \int_{0}^{\infty} \frac{V_{F}^{\frac{p}{2}}}{\sup \left(K\left(V_{F}^{\frac{1}{2}}\right), 1\right)} \leq \text { const. }\|u\|_{p}^{p} .
$$

Therefore, by Barbălat's lemma, we get that $x_{u}$ converges to 0 at infinity.

Next, we establish a similar result to Lemma 3.15:

Lemma 4.4. Let $p \in[1, \infty), u \in L^{p}$ and $x_{u}=(z, y)$ be the trajectory of $\left(S I_{2}\right)_{k_{F}}$ corresponding to $u$ and starting at 0 . Then, we have

$$
\left\|K(y)^{2}\right\|_{p} \leq \text { const. }\|z \sigma(z+u)\|_{p}
$$

with equality if $p=1$.

Proof of Lemma 4.4. Consider the function

$$
Q_{F}(z, y) \stackrel{\text { def }}{=}-z K(y)\left(K(y)^{2}\right)^{p-1}+\int_{0}^{y} K(s)\left(K(y)^{2}\right)^{p-1} \mathrm{~d} s .
$$

Let $\dot{Q}_{F}$ be the time derivative of $Q_{F}$ along $x_{u}$. We have

$$
\dot{Q}_{F}=-|K(y)|^{2 p}+(2 p-1)\left(K(y)^{2}\right)^{p-1} z \sigma(z+u) .
$$

Similarly to Lemma 3.15, we integrate (4.11) between 0 and $T$ for every $T>0$, then we apply Hölder's inequality (for $p>1$ ), and we conclude by letting $T$ goes to $\infty$ and by taking into account Lemma 4.3.

As a corollary, we prove Theorem 2.8 for $p=1$. According to Lemma 4.4, we have

$$
\|z \sigma(z+u)\|_{1}=\left\|K(y)^{2}\right\|_{1} \leq \text { const. }\|y\|_{1} .
$$

Moreover, dividing (4.6) by $V_{F}^{\frac{1}{2}}$ implies that

$$
\left(L_{1}\left(V_{F}^{\frac{1}{2}}\right)\right)+V_{F}^{\frac{1}{2}} \frac{1+|K(y)|}{2 K\left(V_{F}^{\frac{1}{2}}\right)} \inf \left(V_{F}^{\frac{1}{2}}, 1\right) \leq 4 \frac{1+|K(y)|}{K\left(V_{F}^{\frac{1}{2}}\right)}|z| \sigma(3|u|) M(z, y, u)
$$

where $L_{1}$ was defined in (2.15). Since $y \mapsto \frac{y}{K(y)}$ is increasing for $y \geq 0$, we have

$$
\frac{V_{F}^{\frac{1}{2}}}{K\left(V_{F}^{\frac{1}{2}}\right)} \geq \text { const. } \frac{|y|}{|K(y)|}
$$


Then, we deduce from equation (4.12) that

$$
\left(L_{1}\left(V_{F}^{\frac{1}{2}}\right)\right)^{\cdot}+\frac{|y|}{2} \leq 12 K|u| .
$$

After integration, we obtain $\|y\|_{1} \leq$ const. $\|u\|_{1}$. A trajectory of $\left(S I_{2}\right)_{k_{F}}$ starting at 0 and corresponding to the input $u$ is also the trajectory of the linear system given by $\dot{x}=A_{1} x+b_{1} w$ with

$$
A_{1}=\left(\begin{array}{rl}
0 & 1 \\
-1 & -1
\end{array}\right), \quad b_{1}=\left(\begin{array}{ll}
0 & 1
\end{array}\right)^{T}
$$

and with $w=(z+u-\sigma(z+u))-u-y+K(y)$. By (ii) of Definition 1, we get $|w| \leq$ const. $(|z \sigma(z+u)|+|u|+|y|)$. Since $A_{1}$ is Hurwitz, we conclude.

From now on, we assume that $R_{m} \stackrel{\text { def }}{=}\|R\|_{\infty},\left\|V_{F}^{\frac{1}{2}}\right\|_{\infty},\|u\|_{p} \geq C^{2}$. In order to prove Theorem 2.8, we look for establishing the inequality

$$
\int_{0}^{\infty} V_{F}^{\frac{p}{2}} \leq \text { const. } \int_{0}^{\infty} V_{F}^{\frac{p-1}{2}}|u|,
$$

which provides the result together with Hölder's inequality.

Set $k_{m} \stackrel{\text { def }}{=}\left[\frac{\ln \frac{R_{m}}{C}}{\ln 2}\right]$. We consider

$$
\left.\begin{array}{l}
S_{0}=\{t \geq 0, \quad R(z(t), y(t))>C \\
S_{1}=\{t \geq 0, \quad R(z(t), y(t)) \leq C
\end{array}\right\} .
$$

As done before, we approximate $S_{1}$ by a decreasing sequence of open sets $\left(S_{1, \varepsilon}\right)$ such that $\left|S_{1, \varepsilon} / S_{1}\right|$ tends to zero as $\varepsilon$ tends to. On each interval $I^{\prime}$ of $S_{1, \varepsilon}$, we look for

$$
\left.\Delta R\right|_{I^{\prime}}+\text { const. } \int_{I^{\prime}} V_{F}^{\frac{p}{2}} \leq \text { const. } \int_{I^{\prime}} V_{F}^{\frac{p-1}{2}}|u|
$$

This last equation is a direct consequence of the fact that (4.7) can be simply written on $S_{1}$ as

$$
\dot{R}+\text { const. } V_{1}^{\frac{p}{2}} \leq \text { const. } V_{1}^{\frac{p-1}{2}}|u| .
$$

Similarly to the proof of Proposition 3.13, we "slice" up $S_{0}$ according to (3.30) with the obvious modifications. On each interval $I$ of $L_{k}$ or $M_{k, \varepsilon}$ falling into one of the (ci) cases, we look for obtaining the inequality

$$
\left.\Delta R\right|_{I}+\text { const. } \int_{I} V_{F}^{\frac{p}{2}} \leq \text { const. } \int_{I} V_{F}^{\frac{p-1}{2}}|u|
$$

Equation (4.13) is finally obtained by summing up (4.15) on $S_{0}$ and (4.14) on $S_{1}$.

Therefore, it remains to show (4.15) to finish the proof of Theorem 2.8. Let $I=\left(t_{0}, t_{1}\right)$ be an interval of some $L_{k}$ or $M_{k, \varepsilon}$ such that $\left.\Delta R\right|_{I}=\mu \frac{\bar{R}}{2}$ where $\mu= \pm 1,0$ and $\bar{R}=\frac{R_{m}}{2^{k}}$. Let

$$
T \stackrel{\text { def }}{=} t_{1}-t_{0} \text { and } \bar{V} \stackrel{\text { def }}{=} R^{-1}(\bar{R})
$$


We easily get that $R^{-1}\left(\frac{\bar{R}}{2}\right)=\lambda \bar{V}$ with

$$
\left(\frac{1}{2}\right)^{\frac{1}{p}} \leq \lambda \leq\left(\frac{1}{2}\right)^{\frac{1}{p+1}}
$$

Therefore $\left.\Delta V_{F}\right|_{I}=(1-\lambda) \bar{V}$ and we have, for every $t \in I, \lambda \bar{V} \leq V_{F}(t) \leq \bar{V}$. In addition, we get

$$
\frac{1}{\sqrt{2}} T \bar{V}^{\frac{p}{2}} \leq \int_{I} V_{F}^{\frac{p}{2}} \leq T \bar{V}^{\frac{p}{2}}
$$

Consider the following subsets of $I$ :

$$
\begin{aligned}
E_{1} & =\left\{t \in I, \quad|y| \geq \rho V_{F}^{\frac{1}{2}}\right\} \\
E_{2} & =\left\{t \in I, \quad|y|<\rho V_{F}^{\frac{1}{2}}\right\} \\
E & =\left\{t \in E_{2}, \quad|u|>\frac{|z|}{2} \text { a.e. }\right\}
\end{aligned}
$$

We first assume that $\left|E_{1}\right| \geq \rho T$. Then, for $t \in E_{1}$, we have

$$
\frac{1+|K(y)|}{2 K\left(V_{F}^{\frac{1}{2}}\right)} \geq \frac{\rho}{2}
$$

which implies that

$$
\int_{I} \frac{1+|K(y)|}{2 K\left(V_{F}^{\frac{1}{2}}\right)} V_{F}^{\frac{p}{2}} \geq \text { const. } \rho \int_{E_{1}} V_{F}^{\frac{p}{2}} \geq \text { const. } \rho T \bar{V}^{\frac{p}{2}},
$$

and we get (4.15) by integration of (4.9).

We next assume that $\left|E_{1}\right|<\rho T$. Then, $|z|>(1-\rho) V_{F}^{\frac{1}{2}}$ on $E_{2}$. Let $k_{0} \stackrel{\text { def }}{=} \sup _{E_{2}}|K(y)|$. We have $k_{0} \leq K\left(\rho V_{F}^{\frac{1}{2}}\right)$ and the following lemma:

Lemma 4.5. With the above notations, let

$$
U \stackrel{\text { def }}{=}\left\{t \in E_{2}, \quad|K(y)| \geq \frac{k_{0}}{2}\right\} .
$$

Then, either $E_{2}=I$ or $|U|+|E| \geq$ const. $T$.

Proof of Lemma 4.5. If $E_{1}$ is not empty, then $k_{0}=K\left(\rho V_{F}^{\frac{1}{2}}\right)$. Since $E_{2}$ is open, it is the countable union of open intervals. For every interval $J=\left(t, t^{\prime}\right)$ of $E_{2}$, let $U_{J} \stackrel{\text { def }}{=} U \cap J$ and $E_{J} \stackrel{\text { def }}{=} E \cap J$. We have $|K(y(t))|,\left|K\left(y\left(t^{\prime}\right)\right)\right| \leq k_{0}$ with equality at $t$ or at $t^{\prime}$. Similarly to (5.8), we get

$$
\mid \sigma(C)\left(t^{\prime}-t\right)-\text { const. }\left|E_{J}\right| \mid \leq 2 k_{0}
$$

Since the time derivative of $K(y)$ is bounded (by $K_{\sigma}$ ), we have $k_{0} \leq$ const. $\left|U_{J}\right|$. Therefore, we obtain

$$
\left|U_{J}\right|+\left|E_{J}\right| \geq \text { const. }\left(t^{\prime}-t\right) \text {. }
$$


Adding up, we conclude.

According to the previous lemma, three possibilities may occur: $E_{1}$ is empty, $|E| \geq$ const. $T$ or $|U| \geq$ const. $T$. Suppose first, that $|E| \geq$ const. $T$. Then, for a.e. $t \in E$, we have

$$
|u|>\frac{|z|}{2}>\text { const. } V_{F}^{\frac{1}{2}}
$$

We deduce that

$$
\int_{I} V_{F}^{\frac{p-1}{2}}|u| \geq \text { const. } \int_{I} V_{F}^{\frac{p}{2}}
$$

This implies (4.15), except in the case where $\left.\Delta R\right|_{I}=\frac{\bar{R}}{2}$. But we also have

$$
\bar{R} \leq \text { const. } \frac{V_{F}^{\frac{p+1}{2}}}{K\left(V_{F}^{\frac{1}{2}}\right)} \text { and } \bar{V} \leq \text { const. }\left.\Delta V_{F}\right|_{I} \leq \text { const. } T K\left(\bar{V}^{\frac{1}{2}}\right) \bar{V}^{\frac{1}{2}} .
$$

Then, $\left.\Delta R\right|_{I} \leq$ const. $\int_{I} V_{F}^{\frac{p-1}{2}}|u|$ and we conclude this case.

Suppose now that $|U| \geq$ const. $T$. We may also assume that $k_{0}=K\left(\rho V_{F}^{\frac{1}{2}}\right)$. Then

$$
\int_{U} \frac{1+|K(y)|}{2 K\left(V_{F}^{\frac{1}{2}}\right)} V_{F}^{\frac{p}{2}} \geq \text { const. } \frac{K\left(\rho \bar{V}^{\frac{1}{2}}\right)}{K\left(\bar{V}^{\frac{1}{2}}\right)}(1-\lambda) T \bar{V}^{\frac{p}{2}} \geq T \bar{V}^{\frac{p}{2}} .
$$

We easily conclude here.

Finally, suppose that $E_{1}$ is empty. Then, $z$ has a constant $\operatorname{sign}$ on $I$, let say $z>(1-\rho) V_{F}^{\frac{1}{2}}$ on $I$. Equation (4.16) becomes

$$
\mid \sigma(C) T-\text { const. }|E| \mid \leq 2 k_{0} \leq \text { const. } K\left(\rho \bar{V}^{\frac{1}{2}}\right) .
$$

If $|\Delta R|_{I} \mid=\frac{\bar{R}}{2}$, then $\left|\Delta V_{F}\right|_{I} \mid \geq$ const. $\bar{V}$. Thanks to (4.6), we deduce that $\left|\Delta V_{F}\right|_{I} \mid \leq$ const. $k_{0} \bar{V}^{\frac{1}{2}}$, which implies that

$$
T \geq \text { const. } \frac{\bar{V}^{\frac{1}{2}}}{K\left(\rho \bar{V}^{\frac{1}{2}}\right)},
$$

with the last const. independent of $\rho$. Since $K\left(\rho \bar{V}^{\frac{1}{2}}\right)^{2} \leq$ const. $\rho \bar{V}^{\frac{1}{2}}$, we obtain, from (4.17), that $|E| \geq$ const. $T$. That situation was already treated. Therefore, the proof of Theorem 2.8 is finished.

\subsection{Proof of Theorem $\mathbf{2 . 9}$}

Using the change of variables given by (4.3), we rewrite $\left(P S I_{2}\right)_{k_{F}}$ as

$$
\left\{\begin{aligned}
\dot{z} & =K(y)+\frac{-\sigma(z+u)+v_{2}}{K^{\prime}(y)}+v_{1}, \\
\dot{y} & =\frac{-\sigma(z+u)+v_{2}}{K^{\prime}(y)} .
\end{aligned}\right.
$$


We choose $\varepsilon_{0}=\frac{\sigma(1)}{4}$. The time derivative of $V_{F}$ along trajectories of $\left(P S I_{2}\right)_{k_{F}}$ becomes $\dot{V}_{F}+\frac{1+|K(y)|}{2} V_{F}^{\frac{1}{2}} \inf \left(V_{F}^{\frac{1}{2}}, 1\right) \leq 8(1+|K(y)|)|z| \sigma\left(\right.$ const. $\left.\left(|u|+\left|v_{1}\right|+\left|v_{2}\right|\right)\right) M(z, y, u)+$ const. $\left|v_{1}\right||y| N(z, y)$, where

$$
N(z, y) \stackrel{\text { def }}{=} \begin{cases}1, & \text { if } V_{F}<1 \\ 0, & \text { otherwise. }\end{cases}
$$

From the previous equation, we can follow exactly the lines of the proof of Theorem 2.8 only changing $|u|$ by $|u|+\left|v_{1}\right|+\left|v_{2}\right|$ and checking at each step that the extra term $\left|v_{1}\right||y| N(z, y)$ does not alter the computations. The term $L_{p}\left(V_{F}(\bar{x})^{1 / 2}\right)$, appearing in (2.14), comes from the integration of (4.7).

\section{ApPEndix}

We first notice that $\left(S I_{2}\right)$ is controllable. We construct the required sequences assuming that $\sigma$ is the standard saturation function $\sigma_{0}$. The difference with an arbitrary $i S$-function simply consists of having a different set of const. below.

\subsection{Construction of $\left(\boldsymbol{u}^{(1)}\right)$}

We construct a l.a.c. curve $c:[0, \infty) \rightarrow \mathbb{R}^{2}$ such that $c$ is a trajectory of $\left(S I_{2}\right)_{k_{L}}$ corresponding to an piecewise $C^{1}$ input $u$. Then, each input of the sequence $\left(u_{n}^{(1)}\right)$ will be the concatenation of $u_{n}$, the restriction of $u$ to an finite time interval $\left[0, T_{n}\right]$, and the zero input for $t>T_{n}$. There exists a continuous input $\bar{u}$ steering 0 to $\left(0, V_{0}^{\frac{1}{2}}\right)$ with $V_{0}^{\frac{1}{2}} \geq C$ in time $T_{0}>0$ along $\left(S I_{2}\right)_{k_{L}}$. Then, $c$ is defined on $\left[0, T_{0}\right]$ as the resulting trajectory. We take $u=C-z$ until $z=y+2$. In time $T^{\prime}$, we reach the point $x^{\prime}=\left(V^{\prime}, V^{\prime}-2\right)$, with

$$
T^{\prime}=V_{0}^{\frac{1}{2}}+\sqrt{V_{0}-4} \text { and } V^{\prime}=V_{0}^{\frac{1}{2}}-2+\sqrt{V_{0}-4} .
$$

From $x^{\prime}$, we take $u$ to be 0 , until $z=0$. In time $T^{\prime \prime}$, we are, hence, at some point $\left(0,-V^{\prime \prime}\right)$, with $T^{\prime \prime}, V^{\prime \prime}=$ $2 V^{\prime}+o\left(\frac{1}{V^{\prime}}\right)$. By symmetry with respect to $(0,0)$, we repeat the procedure from $\left(0,-V^{\prime \prime}\right)$ and we end up at $\left(0, V_{1}\right)$ for a time $T_{1}$. We clearly have

$$
T_{1}-T_{0}, V_{1}-V_{0}=4 V_{0}^{\frac{1}{2}}+o\left(\frac{1}{V_{0}^{\frac{1}{2}}}\right) .
$$

The above construction is then repeated. We, thus, define two sequences $\left(V_{k}\right)_{k \geq 0}$ and $\left(T_{k}\right)_{k \geq 0}$ such that we have

$$
\text { const. } V_{k}^{\frac{1}{2}} \leq T_{k+1}-T_{k} \leq \text { const. } V_{k}^{\frac{1}{2}} \text {, const. } V_{k}^{\frac{1}{2}} \leq V_{k+1}-V_{k} \leq \text { const. } V_{k}^{\frac{1}{2}} \text {. }
$$

We also obtain that, for $k \geq 0$,

$$
\text { const. } k^{2}+V_{0} \leq V_{k} \leq \text { const. } k^{2}+V_{0} .
$$

It is easy then to get that, for $1 \leq p \leq q$ and $k$ large,

$$
\begin{aligned}
& \text { const. } k^{2 q+1} \leq \int_{T_{k}}^{T_{k+1}}|z|^{q} \leq \text { const. } k^{2 q+1} \text {, const. } k^{q+1} \leq \int_{T_{k}}^{T_{k+1}}|y|^{q} \leq \text { const. } k^{q+1}, \\
& \text { const. } k^{p} \leq \int_{T_{k}}^{T_{k+1}}|u|^{p} \leq \text { const. } k^{p} .
\end{aligned}
$$


For $n \geq 0$, the input $u_{n}^{(1)}$ is defined as $u$ on $\left[0, T_{n}\right]$ and 0 afterwards. If we use $\left(z_{n}^{(1)}, y_{n}^{(1)}\right)$ to denote the resulting trajectory, then we get, for $1 \leq p \leq q \leq \infty$,

$$
\begin{aligned}
\text { const. } n^{\frac{2(q+1)}{q}} & \leq\left\|z_{n}^{(1)}\right\|_{q} \leq \text { const. } n^{\frac{2(q+1)}{q}} \\
\text { const. } n^{\frac{q+2}{q}} & \leq\left\|y_{n}^{(1)}\right\|_{q} \leq \text { const. } n^{\frac{q+2}{q}} \\
\text { const. } n^{\frac{p+1}{p}} & \leq\left\|u_{n}^{(1)}\right\|_{p} \leq \text { const. } n^{\frac{p+1}{p}} .
\end{aligned}
$$

\subsection{Construction of $\left(\boldsymbol{u}^{(2)}\right)$}

We already know that the shape found in Proposition 3.7 is the profile (3) in Theorem 2.5, thanks to Remark 2.4. But, even though the $L^{p}$-norms of the inputs $\bar{u}_{n}$ involved to obtain the preceding result can be chosen increasing to $\infty$, their $L^{\infty}$-norms are, of course, very small. For $n \geq 0$, the input $u_{n}^{(2)}$ is simply obtained as the concatenation of an input $v_{n}$ and an input $\bar{u}_{n_{k}}$ given as follows: $v_{n}$ is defined on some $\left[0, T_{v_{n}}\right]$ with large $L^{\infty}$-norm and such that the resulting trajectory reaches the origin at time $T_{v_{n}} ; \bar{u}_{n_{k}}$ is chosen such that its $L^{p}$-norm is bigger than the $L^{p}$-norm of $v_{n}$. Therefore, the sequence $\left(u_{n}^{(2)}\right)$ completes the proof that, the shape found in Proposition 3.7, is the profile (3) in Theorem 2.5 and the $u_{n}^{(2)}$ have arbitrarily large $L^{\infty}$-norm.

\subsection{Proof of Proposition 3.3}

We use $y_{\max }$ and $z_{\max }$ to denote $\|y\|_{\infty,[0, T]}$ and $\|z\|_{\infty,[0, T]}$ respectively.

We start with the argument for $(a)$. We pick $\left[t_{0}, t_{1}\right] \in[0, T]$ such that we have, for $t \in\left[t_{0}, t_{1}\right]$,

$$
\left|y\left(t_{0}\right)\right|=\frac{y_{\max }}{2} \leq|y(t)| \leq\left|y\left(t_{1}\right)\right|=y_{\max } .
$$

Recall that $\frac{y_{\max }}{2}$ is in the range of $y$ since the initial state is zero. Without loss of generality, we consider the case $y(t) \geq 0$ on $\left[t_{0}, t_{1}\right]$. Let $L \stackrel{\text { def }}{=} t_{1}-t_{0}$. Then,

$$
\frac{y_{\max }}{2}=\left.\Delta y\right|_{t_{0}} ^{t_{1}}=-\int_{t_{0}}^{t_{1}} \sigma(z+u) \leq K L,
$$

and

$$
2 z_{\max } \geq\left.\Delta z\right|_{t_{0}} ^{t_{1}}=\int_{t_{0}}^{t_{1}} y+\left.\Delta y\right|_{t_{0}} ^{t_{1}} \geq \frac{y_{\max }}{2}(L+1)
$$

The two previous equations imply that $z_{\max } \geq \frac{y_{\max }^{2}}{8 K}$.

As for item $(b)$, we pick $\left[t_{0}, t_{1}\right] \in[0, T]$ such that we have, for $t \in\left[t_{0}, t_{1}\right]$,

$$
W\left(t_{0}\right)=\frac{W_{\max }}{2} \leq W(t) \leq W\left(t_{1}\right)=W_{\max },
$$

where $W$ is given by (3.3) and $W_{\max }=\|W\|_{\infty,[0, T]}$. Note that $\sigma_{m} \leq \frac{W_{\max }}{z_{\max }} \leq$ const. We may assume that, for every $t \in\left[t_{0}, t_{1}\right],|y(t)|<\sqrt{\frac{W_{\max }}{2}}$. This clearly implies that, for every $t \in\left[t_{0}, t_{1}\right]$,

$$
|z(t)| \geq \frac{W_{\max }}{4 \sigma_{M}}
$$


in which case $z$ has a constant sign on $\left[t_{0}, t_{1}\right]$. Assume now that $W_{\max }<C$. Then (3.5) follows immediately since $\|u\|_{p} \geq C$. For the rest of the argument, $W_{\max } \geq C$. Consider the two following subsets of $\left[t_{0}, t_{1}\right]$ given by

$$
\begin{aligned}
& E_{1}=\left\{t \in\left[t_{0}, t_{1}\right],|u(t)|<\frac{|z(t)|}{3} \text { a.e. }\right\} \\
& E_{2}=\left\{t \in\left[t_{0}, t_{1}\right],|u(t)| \geq \frac{|z(t)|}{3} \text { a.e. }\right\}
\end{aligned}
$$

We derive $W$ along the trajectory of $\left(S I_{2}\right)_{k_{L}}$ corresponding to $u$ and get

$$
\dot{W}=-\left.y \Delta \sigma\right|_{z} ^{z+u}-\sigma(z) \sigma(z+u)=-\left.\dot{z} \Delta \sigma\right|_{z} ^{z+u}-\sigma^{2}(z+u)
$$

Note that $|\dot{z}| \leq \sqrt{\frac{W_{\max }}{2}}+K$ and $|\Delta \sigma|_{z}^{z+u} \mid \leq \delta_{C}$ on $E_{1}$. We integrate (5.6) between $t_{0}$ and $t_{1}$ and since $\int_{t_{0}}^{t_{1}} \dot{W}=\int_{E_{1}} \dot{W}+\int_{E_{2}} \dot{W}$, we get

$$
\sigma_{m}^{2}\left(L-\left|E_{2}\right|\right)+\frac{W_{\max }}{2} \leq\left(L-\left|E_{2}\right|\right) \delta_{C}\left(\sqrt{\frac{W_{\max }}{2}}+K\right)+\left(\sqrt{\frac{W_{\max }}{2}}+K\right) 2 K\left|E_{2}\right| .
$$

The above equation can be written as

$$
\sigma_{m}^{2} L+\frac{W_{\max }}{2} \leq L \delta_{C} \sqrt{2 W_{\max }}+3 K\left|E_{2}\right|\left(\sqrt{\frac{W_{\max }}{2}}+K\right)
$$

On the other hand, we have

$$
\left.\Delta y\right|_{t_{0}} ^{t_{1}}=-\int_{t_{0}}^{t_{1}} \sigma(z)+\left.\int_{t_{0}}^{t_{1}} \Delta \sigma\right|_{z} ^{z+u}
$$

which implies that

$$
\sigma_{m} L \leq \int_{t_{0}}^{t_{1}} \sigma(z) \leq-\left.\Delta y\right|_{t_{0}} ^{t_{1}}+\left.\int_{E_{1}} \Delta \sigma\right|_{z} ^{z+u}+\left.\int_{E_{2}} \Delta \sigma\right|_{z} ^{z+u}
$$

Using the definition of $E_{1}$ and $E_{2}$, we get

$$
\sigma_{m} L \leq 2 y_{\max }+\left(L-\left|E_{2}\right|\right) \delta_{C}+2 K\left|E_{2}\right|
$$

We thus obtain

$$
L \leq \frac{4}{\sigma_{m}} \sqrt{\frac{W_{\max }}{2}}+\frac{6 K}{\sigma_{m}}\left|E_{2}\right|
$$

Equations (5.7) and (5.10) give $\sqrt{W_{\max }} \leq$ const. $\left|E_{2}\right|$ which, in turn, implies that for $p$ finite

$$
\|u\|_{p,[0, T]}^{p} \geq \int_{E_{2}}|u|^{p} \geq W_{\max }^{p+\frac{1}{2}},
$$

and $\mid\left\{t \in\left[t_{0}, t_{1}\right], \quad|u(t)| \geq\right.$ const. $W_{\max }$ a.e. $\} \mid>$ const. $L$. The proof of Proposition 3.3 is finished. 


\subsection{Proof of Lemma 3.5}

If $|z(t)|=M+C$ for $t \in\left[t_{0}, t_{1}\right]$, then $|\dot{z}| \geq|y|-K \geq \frac{\sqrt{\lambda}}{4} M$. Thanks to Remark 3.2, the zeros of $|z|-(M+C)$ are isolated. Then Lemma 3.5 simply says, unless trivial cases, there is an odd number of zeros of $|z|-(M+C)$ such that the variation of $W$ between the smallest and the largest zeros is larger than $\frac{\lambda}{16} M^{2}$. We do so by examining several cases.

Assume that $\left|z\left(t_{0}\right)\right|>M+C$ and $\left|z\left(t_{1}\right)\right|>M+C$. In addition, suppose that

$$
\text { for all } t \in\left[t_{0}, t_{1}\right],|z(t)| \geq M+C \text {. }
$$

Then, we have $|\dot{y}| \geq \frac{3}{4} \sigma_{m}$. It implies that

$$
\frac{3}{4} \sigma_{m}\left(t_{1}-t_{0}\right) \leq|\Delta \sigma|_{t_{0}}^{t_{1}} y \mid \leq 2 y_{\max }
$$

We deduce that $L=t_{1}-t_{0} \leq \frac{8 y_{\max }}{3 \sigma_{m}}$. By integrating (5.6) and using (3.6), we get

$$
\frac{\lambda}{2} M^{2}=\left.\Delta W\right|_{t_{0}} ^{t_{1}} \leq \int_{t_{0}}^{t_{1}}(|y|+K)|\Delta \sigma|_{|z|}^{|z|+M} \mid-\int_{t_{0}}^{t_{1}} \sigma^{2}(|z|-M) .
$$

By using (3.6), we have

It implies that

$$
\int_{t_{0}}^{t_{1}}(|y|+K)|\Delta \sigma|_{|z|}^{|z|+M} \mid \leq 2 \delta_{C} L y_{\max }
$$

$$
\frac{\lambda}{2} M^{2} \leq \delta_{C} \text { const. } \lambda M^{2}-\left(\frac{3}{4} \sigma_{m}\right)^{2} L
$$

which is impossible. Then (5.11) cannot hold. Therefore, let $t^{1} \in\left[t_{0}, t_{1}\right]$ such that $\left|z\left(t^{1}\right)\right|=M+C$ and $|z| \geq M+C$ on $\left[t_{0}, t_{2}\right]$. Then $\left.\Delta W\right|_{t^{1}} ^{t_{1}} \geq \frac{\lambda}{4} M^{2}$. By reproducing the previous argument on $\left[t^{1}, t_{1}\right]$, we can find $t^{2} \in\left[t^{1}, t_{1}\right]$ such that $\left|z\left(t^{2}\right)\right|=M+C$ and $\left.\Delta W\right|_{t^{1}} ^{t^{2}} \geq \frac{\lambda}{8} M^{2}$. Again, unless $\lambda$ is bounded, we can get a third zero of $|z|-(M+C)$ with the required properties. Going on in the same fashion, we prove the conclusions of Lemma 3.5.

Let us study now the case where $\left|z\left(t_{0}\right)\right|<M+C$ and $\left|z\left(t_{1}\right)\right|<M+C$. Suppose that

$$
\text { for all } t \in\left[t_{0}, t_{1}\right],|z(t)| \leq M+C \text {. }
$$

Then, for all $t \in\left[t_{0}, t_{1}\right]$, we have $|y(t)| \geq \frac{\sqrt{\lambda}}{4} M$. Hence,

$$
2(M+C) \geq|\Delta z|_{t_{0}}^{t_{1}} \mid \geq L \frac{\sqrt{\lambda}}{8} M .
$$

On the other hand, we also have $\frac{\lambda}{2} M^{2}=\left.\Delta W\right|_{t_{0}} ^{t_{1}} \leq$ const. $\sqrt{\lambda} M L$. We finally get that $M$ is bounded by a constant independent of $u$, which is impossible. Therefore (5.12) cannot hold and there exists $t_{0}^{\prime} \in\left[t_{0}, t_{1}\right]$ such that $\left|z\left(t_{0}^{\prime}\right)\right|>M+C$. Then, if $t_{2}$ is the first time after $t_{0}$ such that $\left|z\left(t_{2}\right)\right|=M+C$ and $t_{3}$ is the first time before $t_{1}$ such that $\left|z\left(t_{3}\right)\right|=M+C$, we have that $\left.\Delta W\right|_{t_{2}} ^{t_{3}} \geq \frac{\lambda}{8} M^{2}$. Again, unless we are done, we can get a third zero for $|z|-(M+C)$ with the required properties.

All the other cases reduce to those studied above and Lemma 3.5 is thus proved. 


\subsection{Proof of Lemma 3.6}

On $I_{2 l-1},|\dot{y}| \geq \frac{3}{4} \sigma_{m}$ and $\left|y\left(t^{j}\right)\right| \geq$ const. $M$. Therefore there exist $t^{i}<t^{f} \in I_{2 l-1}$ such that $|y|>\sigma_{M}$ on $\left[t^{2 l-1}, t^{i}[\right.$ and $\left.] t^{f}, t^{2 l-1}\right]$ and $|y| \leq \sigma_{M}$ on $\left[t^{i}, t^{f}\right]$. We get $|\dot{z}|>0$ on $\left[t^{2 l-1}, t^{i}[\right.$ and $\left.] t^{f}, t^{2 l}\right]$. We may assume that $z>0$ on $I_{2 l-1}$. Let

Then

$$
z_{m} \stackrel{\text { def }}{=} \min \left(z\left(t^{2 l-1}\right), z\left(t^{i}\right) \text { and } z_{m}^{\prime}=\max \left(z\left(t^{2 l-1}\right), z\left(t^{i}\right)\right)\right.
$$

$$
\begin{aligned}
\left.\Delta W\right|_{t^{2 l-1}} ^{t^{i}} & =\int_{t^{2 l-1}}^{t^{i}}-\left(\left.\dot{z} \Delta \sigma\right|_{z} ^{z+u}+\sigma^{2}(z+u)\right) \mathrm{d} t=-\left.\int_{z\left(t^{2 l-1}\right)}^{z\left(t^{i}\right)} \Delta \sigma\right|_{z} ^{z+u} \mathrm{~d} z-\int_{t^{2 l-1}}^{t^{i}} \sigma^{2}(z+u) \mathrm{d} t \\
& \leq \int_{z_{m}}^{z_{m}^{\prime}}|\Delta \sigma|_{z}^{z+u} \mid \mathrm{d} z-\int_{t^{2 l-1}}^{t^{i}} \sigma^{2}(z-M) \mathrm{d} t
\end{aligned}
$$

Since $\sigma$ is increasing and globally Lipschitz, it has a non negative derivative function $\sigma^{\prime}$ locally essentially bounded. Define

$$
J \stackrel{\text { def }}{=} \int_{z_{m}}^{z_{m}^{\prime}}|\Delta \sigma|_{z}^{z+u} \mid \mathrm{d} z
$$

Then, we have

$$
J \leq \int_{z_{m}}^{z_{m}^{\prime}} \int_{z-M}^{z+M} \sigma^{\prime}(s) \mathrm{d} s \mathrm{~d} z=\iint_{\substack{z m \leq z \leq z_{m}^{\prime} \\ z-M \leq s \leq z+M}} \sigma^{\prime}(s) \mathrm{d} s \mathrm{~d} z \leq \iint_{z-M \leq s \leq z+M}{ }_{\substack{c \leq z \leq z_{m}^{\prime}+M \\ z-M \leq z+m}} \sigma^{\prime}(s) \mathrm{d} s \mathrm{~d} z
$$

By Fubini's theorem, we can permute the order of integration in $J$ and we obtain

$$
J \leq \iint_{C \leq s \leq \operatorname{const.~} \lambda M^{2}} \sigma^{\prime}(s) \mathrm{d} s \mathrm{~d} z \leq \int_{C}^{\text {const. } \lambda M^{2}} \sigma^{\prime}(s) \int_{\max \left(z_{m}, s-M\right)}^{\min \left(z_{m}^{\prime}, s+M\right)} \mathrm{d} z \mathrm{~d} s \leq\left. 2 M \Delta \sigma\right|_{C} ^{\text {const. } \lambda M^{2}} \leq 2 M \delta_{C}
$$

Then, we get

In addition, we have

$$
\left.\Delta W\right|_{t^{2 l-1}} ^{t^{i}} \leq 2 M \delta_{C}-\frac{\sigma_{m}^{2}}{2}\left(t^{i}-t^{2 l-1}\right)
$$

in which case

$$
\sigma_{M}\left(t^{i}-t^{2 l-1}\right) \geq|\Delta y|_{t^{2 l-1}}^{t^{i}} \mid \geq \text { const. } \sqrt{\lambda} M
$$

$$
\left.\Delta W\right|_{t^{2 l-1}} ^{t^{i}} \leq 2 M \delta_{C}-\text { const. } \sqrt{\lambda} M \leq- \text { const. } \sqrt{\lambda} M
$$

Moreover, since $\sigma_{M}\left(t^{f}-t^{i}\right) \geq|\Delta y|_{t^{i}}^{t^{f}} \mid=2 \sigma_{M}$, we have

$$
\left.\Delta W\right|_{t^{i}} ^{t^{f}} \leq \sigma_{M} \delta_{C}-\left(\frac{3 \sigma_{m}}{4}\right)^{2}\left(t^{f}-t^{i}\right) \leq 0
$$

Then, we obtain $\Delta W_{2 l-1} \leq-$ const. $\sqrt{\lambda} M$. On $I_{2 l},|y| \geq$ const. $\sqrt{\lambda} M$, which implies that

$$
\Delta W_{2 l} \leq \text { const. } \sqrt{\lambda} M\left(t^{2 l+1}-t^{2 l}\right) \leq \text { const. }\left|\int_{I_{2 l}} y\right| \leq \text { const. }|\Delta z|_{t^{2 l}}^{t^{2 l+1}} \mid \leq \text { const. } M .
$$

Adding the appropriate inequalities, we get the conclusion of Lemma 3.6. 


\subsection{Proof of Lemma $\mathbf{3 . 1 2}$}

We start with $(J 2)$. We assume that $z \geq 0$.If $u+y<0$, then $|u|>y$, in which case we have

$$
|2 \dot{z} \Delta \sigma|_{z-\theta y}^{z+u}+\left.\sigma(z-y) \Delta \sigma\right|_{z-y} ^{z+u} \mid \leq \text { const. }|u|^{p}
$$

If $u+y \geq 0$, then $\left.\Delta \sigma\right|_{z-y} ^{z+u} \geq 0$, since $\sigma$ is increasing. We have to distinguish two subcases, whether $u+\theta y \geq 0$ or not. If $u+\theta y \geq 0$, then $\left.\Delta \sigma\right|_{z-\theta y} ^{z+u} \geq 0$. Then,

$$
-\left(\left.2 \dot{z} \Delta \sigma\right|_{z-\theta y} ^{z+u}+\left.\sigma(z-y) \Delta \sigma\right|_{z-y} ^{z+u}\right) \leq 0,
$$

and (J2) is immediate. If $u+\theta y<0$, then $|u|>\theta y$ and we get equation (5.14).

We turn to the case where $z \leq 0$. If $u+\theta y<0$, then $|u|>\theta y$, and equation (5.14) follows readily. Assume, then, that $u+\theta y \geq 0$. We also have that $u+y \geq(1-\theta) y \geq \frac{C}{2}$. We distinguish two subcases, whether $z+u \geq-C$ or not. If $z+u \geq-C$, then $u>|z|-C \geq y$ and equation (5.14) follows. If $z+u<-C$, then $|\Delta \sigma|_{z-y}^{z+u} \mid \leq \delta_{C}$ and

and we get $(J 2)$.

$$
-\left(\left.2 \dot{z} \Delta \sigma\right|_{z-\theta y} ^{z+u}+\left.\sigma(z-y) \Delta \sigma\right|_{z-y} ^{z+u}\right) \leq \text { const. } \delta_{C}
$$

For $(J 3)$, we may simply assume that $z \geq 0$. We have

$$
J_{3} \leq-\frac{\sigma_{m}^{2}}{4}\left|I / I^{\prime}\right|+K^{2}\left|E^{\prime}\right|,
$$

where $E^{\prime}=\left\{t \in I / I^{\prime},|u(t)| \geq \frac{z(t)}{3}\right.$ a.e. $\}$. Since $z \geq 1$ on $I / I^{\prime}$, we get (J3).

It remains to show $(J 1)$. We simply assume that $z \geq 0$. Set $z_{1}^{\prime}=z\left(t_{1}^{\prime}\right), z_{1}=z\left(t_{1}\right)$. Since $\dot{z} \geq C$ on $I / I^{\prime}$, we can take $z$ as a new parameter instead of the time $t$ for functions defined on $I / I^{\prime}$. In particular, we can consider $y$ as a function of $z$ on $\left[z_{0}, z_{1}\right]$. In addition, we rewrite (3.15) as

$$
\begin{aligned}
J_{1} & =-\left.\int_{z_{1}^{\prime}}^{z_{1}} \Delta \sigma\right|_{z} ^{z-\theta y} \mathrm{~d} z-\left.\int_{z_{1}^{\prime}}^{z_{1}} \Delta \sigma\right|_{z-y} ^{z-\theta y} \mathrm{~d} z \\
& =\iint_{\substack{z-\theta y(z) \leq s \leq z \\
z_{1}^{\prime} \leq z \leq z_{1}}} \sigma^{\prime}(s) \mathrm{d} s \mathrm{~d} z-\iint_{\substack{z-y(z) \leq s \leq z-\theta y(z) \\
z_{1}^{\prime} \leq z \leq z_{1}}} \sigma^{\prime}(s) \mathrm{d} s \mathrm{~d} z .
\end{aligned}
$$

Note that $z-\theta y$ and $z-y$ can be reparameterized as functions of $z \in\left[z_{0}-y\left(z_{0}\right), z_{1}\right]$ and they verify

$$
\frac{1}{2} \leq \frac{\mathrm{d}(z-\theta y)}{\mathrm{d} z}, \frac{\mathrm{d}(z-y)}{\mathrm{d} z} \leq 2
$$

Therefore, we can define, for $s \in\left[z_{0}-y\left(z_{0}\right), z_{1}\right]$, two functions $Z(s)$ and $\bar{Z}(s)$ as follows

$$
\begin{array}{rll}
Z(s)=z_{0} & \text { for } & s \in\left[z_{0}-y\left(z_{0}\right), z_{0}\right] \\
Z(s)-\theta y(Z(s))=s & \text { for } & s \in\left[z_{0}, z_{1}-\theta y\left(z_{1}\right)\right] \\
Z(s)=z_{1} & \text { for } & s \in\left[z_{1}-\theta y\left(z_{1}\right), z_{1}\right]
\end{array}
$$

and

$$
\begin{array}{rll}
\bar{Z}(s)-y(\bar{Z}(s))=s & \text { for } & s \in\left[z_{0}-y\left(z_{0}\right), z_{1}-y\left(z_{1}\right)\right] \\
\bar{Z}(s)=z_{1} & \text { for } & s \in\left[z_{1}-y\left(z_{1}\right), z_{1}\right] .
\end{array}
$$


By Fubini's theorem, we can permute the order of integration in (5.15) and we obtain

$$
J_{1}=J_{1}^{\prime}+J_{2}^{\prime}+J_{3}^{\prime}+J_{4}^{\prime},
$$

where

$$
\begin{aligned}
J_{1}^{\prime} & =\int_{z_{0}}^{z_{1}-y\left(z_{1}\right)}(2 Z(s)-s-\bar{Z}(s)) \sigma^{\prime}(s) \mathrm{d} s, \\
J_{2}^{\prime} & =\int_{z_{0}-y\left(z_{0}\right)}^{z_{0}-\theta y\left(z_{0}\right)}(Z(s)-\bar{Z}(s)) \sigma^{\prime}(s) \mathrm{d} s \\
J_{3}^{\prime} & =\int_{z_{0}-\theta y\left(z_{0}\right)}^{z_{0}}\left(2 Z(s)-z_{0}-\bar{Z}(s)\right) \sigma^{\prime}(s) \mathrm{d} s, \\
J_{4}^{\prime} & =\int_{z_{1}-y\left(z_{1}\right)}^{z_{1}}(Z(s)-s) \sigma^{\prime}(s) \mathrm{d} s .
\end{aligned}
$$

Since $Z(s) \leq \bar{Z}(s)$ and $Z(s) \leq s$ on $s \in\left[z_{0}-y\left(z_{0}\right), z_{0}\right]$, we have $J_{2}^{\prime} \leq 0$ and $J_{3}^{\prime} \leq 0$. In addition, we have

$$
J_{4}^{\prime}=\int_{z_{1}-y\left(z_{1}\right)}^{z_{1}}(\sigma(z)-\sigma(z-\theta y)) \mathrm{d} z \leq 2 \sup \left(y\left(t_{1}\right), C\right) \delta_{C},
$$

since $z>z-\theta y \geq 2 C$ for $s \in\left[z_{1}-y\left(z_{1}\right), z_{1}\right]$. As for $J_{1}^{\prime}$, we can write it as follows

$$
J_{1}^{\prime}=\int_{z_{0}}^{z_{1}-y\left(z_{1}\right)}(2 \theta y(Z(s))-y(\bar{Z}(s))) \sigma^{\prime}(s) \mathrm{d} s .
$$

We get $J_{1}^{\prime} \leq 0$ as a consequence of the next lemma:

Lemma 5.1. For $s \in\left[z_{0}, z_{1}-y\left(z_{1}\right)\right]$, we have $2 \theta y(Z(s))-y(\bar{Z}(s)) \leq 0$.

Proof of Lemma 5.1. We have

$$
2 \theta y(Z(s))-y(\bar{Z}(s))=\left.2 \theta \Delta y\right|_{Z(s)} ^{\bar{Z}(s)}-(1-2 \theta) y(\bar{Z}(s)) \leq-(1-2 \theta) C+\left.2 \theta \Delta y\right|_{Z(s)} ^{\bar{Z}(s)} .
$$

On the other hand, we have

$$
\left.\left|\Delta y^{2}\right|_{Z(s)}^{\bar{Z}(s)}|\leq 2| \int_{Z(s)}^{\bar{Z}(s)} \frac{y \mathrm{~d} y}{\mathrm{~d} z} \mathrm{~d} z\left|\leq 2 \int_{Z(s)}^{\bar{Z}(s)} \frac{|y \sigma(z+u)|}{y-\sigma(z+u)} \mathrm{d} z \leq \frac{2 K}{1-\frac{K}{C}}\right| \Delta z\right|_{Z(s)} ^{\bar{Z}(s)} \mid \leq \frac{2 C K}{C-K} y(\bar{Z}(s)) .
$$

We thus obtain

$$
|\Delta y|_{Z(s)}^{\bar{Z}(s)} \mid \leq \frac{2 C K}{C-K} \frac{y(\bar{Z}(s))}{y(Z(s))+y(\bar{Z}(s))} \leq \frac{2 C K}{C-K} .
$$

Then, $2 \theta y(Z(s))-y(\bar{Z}(s)) \leq-(1-2 \theta) C+\frac{2 C K}{C-K}=0$.

\subsection{Proof of Lemma 3.15}

Consider the function

$$
Q(z, y) \stackrel{\text { def }}{=}-z y|y|^{2 p-2}+\frac{|y|^{2 p}}{p} .
$$

If we use $\dot{Q}$ to denote the time derivative of $Q$ along $x_{u}$, we get

$$
\dot{Q}=-|y|^{2 p}+(2 p-1)|y|^{2 p-2} z \sigma(z+u) .
$$


For every $T>0$, we integrate the above inequality on $[0, T]$ and get

$$
Q(T)+\int_{0}^{T}|y|^{2 p} \leq \text { const. } \int_{0}^{T}\left(|y|^{2 p}\right)^{\frac{p-1}{p}}|z \sigma(z+u)| .
$$

By applying Hölder's inequality for $p>1$, we get

$$
Q(T)+\int_{0}^{T}|y|^{2 p} \leq \mathrm{const.}\left(\int_{0}^{T}|y|^{2 p}\right)^{\frac{p-1}{p}}\|z \sigma(z+u)\|_{p} .
$$

By letting $T$ tend to infinity and thanks to Remark 3.8, we get the result.

\subsection{Proof of Lemma 3.16}

The two previous lemmas clearly imply that

$$
\|V\|_{p} \leq \text { const. }\left(\|V \sigma(z+u)\|_{p}+\|u\|_{p}\right) .
$$

Therefore, we have, by using Proposition 3.7,

$$
\begin{aligned}
\int_{0}^{\infty} V^{p} & \leq \text { const. }\left(\int_{0}^{\infty} V^{p}|\sigma(z+u)|^{p}+\|u\|_{p}^{p}\right) \leq \text { const. }\left(\int_{E_{R}} V^{p}|\sigma(z+u)|^{p}+\int_{\mathbb{R}^{+} / E_{R}} V^{p}\left|\sigma(z+u)+\|u\|_{p}^{p}\right|^{p}\right) \\
& \leq \text { const. }\left(\int_{E_{R}} V^{p}|\sigma(z+u)|^{p}+\text { const. } R^{p}\|\sigma(z+u)\|_{p}^{p}+\|u\|_{p}^{p}\right) \leq \text { const. } \int_{E_{R}} V^{p}+\text { const. } R^{p}\|u\|_{p}^{p} .
\end{aligned}
$$

From the last equation, we conclude.

\subsection{Proof of Lemma $\mathbf{3 . 1 8}$}

The set $F_{i}$ is an interval $\left[t^{\prime}, t^{\prime \prime}\right]$ such that $\frac{1}{2} \leq t^{\prime}-t_{i} \leq \frac{3}{2}$ and $\frac{1}{2} \leq t_{i+1}-t^{\prime \prime} \leq \frac{3}{2}$. Set $S_{i}=\left[t_{i}, t_{i+1}\right] / F_{i}$. We have $1 \leq\left|S_{i}\right| \leq 3$ and $|y| \geq \frac{\bar{V}^{\frac{1}{2}}}{2}$ on $S_{i}$. Then,

$$
\begin{aligned}
\int_{S_{i}} \dot{V} & =\int_{S_{i} \cap E_{i}} \dot{V}+\int_{S_{i} \cap E_{i}^{\prime}} \dot{V} \leq \text { const. }\left|S_{i} \cap E_{i}\right| \bar{V}^{\frac{1}{2}}-\left|S_{i} \cap E_{i}^{\prime}\right| \frac{\sigma_{m}^{2}+\bar{V}^{\frac{1}{2}}}{2} \\
& \leq-\frac{1}{4} \bar{V}^{\frac{1}{2}}+\text { const. } \bar{V}^{\frac{1}{2}}\left|S_{i} \cap E_{i}\right| .
\end{aligned}
$$

On $F_{i}$, using the estimates obtained in Lemma 3.12 of case $(B 1)$, we get

$$
\int_{F_{i}} \dot{V} \leq-\frac{\sigma_{m}^{2}}{4} \bar{V}^{\frac{1}{2}}+\text { const. }\left|F_{i} \cap E_{i}\right|+2 \int_{F_{i}^{\prime}}|\dot{z}||\Delta \sigma|_{z+u}^{z-\theta y} \mid .
$$

We also have

$$
\int_{F_{i}^{\prime} / G_{i}}|\dot{z}||\Delta \sigma|_{z+u}^{z-\theta y}\left|\leq \int_{F_{i}^{\prime}}\right| \dot{z}|\Delta \sigma|_{z-\rho \bar{V}^{\frac{1}{2}}}^{z} \leq\left.\int_{F_{i}}|\dot{z}| \Delta \sigma\right|_{z-\rho \bar{V}^{\frac{1}{2}}} ^{z}
$$

We need the next lemma to complete the proof of Lemma 3.18:

Lemma 5.2. With the previous notations, we have

$$
\left.\int_{F_{i}}|\dot{z}| \Delta \sigma\right|_{z-\rho \bar{V}^{\frac{1}{2}}} ^{z} \leq \text { const. } \delta_{\bar{V}^{\frac{1}{2}}}\left(\left|E_{i}\right|+\bar{V}^{\frac{1}{2}}\right) .
$$


Proof of Lemma 5.2. Define $L_{i}=\left[t^{\prime}, \bar{t}^{\prime}\right] \cup\left[\bar{t}^{\prime \prime}, t^{\prime \prime}\right]$ and $M_{i}=\left(\bar{t}^{\prime}, \bar{t}^{\prime \prime}\right)$ in such a way that $|y|>\sigma_{M}+1$ on $L_{i}$ and $\left|y\left(\bar{t}^{\prime}\right)\right|=\left|y\left(\bar{t}^{\prime \prime}\right)\right|=\sigma_{M}+1$. Assume first that

$$
\left|M_{i} \cap E_{i}\right|<\frac{\sigma_{m}}{4 K}\left|L_{i}\right| .
$$

Let us show that $\left|M_{i}\right|$ is bounded, in which case $|y|$ remains also bounded. For that purpose, consider the following equation

It implies that

$$
\left.\Delta y\right|_{\bar{t}^{\prime}} ^{\bar{t}^{\prime \prime}} \leq-\int_{M_{i}} \sigma(z)+\int_{M_{i}}|\Delta \sigma|_{z+u}^{z} \mid .
$$

$$
\sigma_{m}\left|M_{i}\right| \leq 2\left(\sigma_{M}+1\right)+\delta_{C}\left|M_{i}\right|+2 K\left|M_{i} \cap E_{i}\right|
$$

Then, by using (5.17), we at once get that $\left|M_{i}\right|$ is bounded. Therefore, we have

$$
\left.\int_{M_{i}}|\dot{z}| \Delta \sigma\right|_{z-\rho \bar{V} \frac{1}{2}} ^{z} \leq \text { const. } \delta_{\bar{V} \frac{1}{2}}
$$

Assume now that $\left|M_{i} \cap E_{i}\right| \geq \frac{\sigma_{m}}{4 K}\left|L_{i}\right|$. Then, $\left|M_{i}\right| \leq$ const. $\left|E_{i}\right|$, in which case we obtain

$$
\left.\int_{M_{i}}|\dot{z}| \Delta \sigma\right|_{z-\rho \bar{V}^{\frac{1}{2}}} ^{z} \leq \text { const. } \delta_{\bar{V}^{\frac{1}{2}}}\left|E_{i}\right| \text {. }
$$

Finally, we have

$$
\left.\int_{L_{i}}|\dot{z}| \Delta \sigma\right|_{z-\rho \bar{V}^{\frac{1}{2}}} ^{z} \leq\left. 3 \int_{\bar{t}^{\prime}}^{\bar{t}^{\prime \prime}} \Delta \sigma\right|_{z-\rho \bar{V}^{\frac{1}{2}}} ^{z} \leq \iint_{\substack{z\left(\bar{t}^{\prime}\right) \leq z \leq z\left(\bar{t}^{\prime \prime}\right) \\ z-\rho \bar{V} \frac{1}{2} \leq s \leq z}} \sigma^{\prime}(s) \mathrm{d} s \mathrm{~d} z \leq\left.\rho \bar{V}^{\frac{1}{2}} \Delta \sigma\right|_{z\left(\bar{t}^{\prime}\right)-\rho \bar{V}^{\frac{1}{2}}} ^{z\left(\bar{t}^{\prime \prime}\right)} \leq \text { const. } \delta_{\bar{V}^{\frac{1}{2}} \bar{V}^{\frac{1}{2}}}
$$

We also have $\int_{G_{i}}|\dot{z}||\Delta \sigma|_{z+u}^{z-\theta y} \mid \leq$ const. $\left|G_{i}\right| \bar{V}^{\frac{1}{2}}$. Adding up all the inequalities, we get (3.39).

\subsection{Proof of Lemma 3.19}

Define $U_{i}=\left\{t \in E_{i}, \quad|z| \geq \frac{\bar{V}^{\frac{1}{2}}}{2 \sigma_{M}}\right\}$. Then, we clearly have

$$
\int_{U_{i}}|z|^{p} \geq \text { const. }\left|U_{i}\right| \bar{V}^{\frac{p}{2}}
$$

We may assume that $\left|U_{i}\right|<\rho\left|E_{i}\right|$, otherwise we are done. Moreover, since $E_{i} / U_{i}$ is included in $S_{i},\left|E_{i}\right|$ is bounded. Recall that $\left|E_{i} / U_{i}\right| \geq \frac{\rho}{2}$ and $|y| \geq \bar{V}^{\frac{1}{2}}$ on $E_{i} / U_{i}$. In addition, we have

$$
|z(t)| \geq \bar{V}^{\frac{1}{2}}\left|t-t_{s}\right|
$$

where $s=i$ or $i+1$. The set $E_{i} / U_{i}$ contains a subset $E_{i}^{\prime \prime}$ of measure larger than $\frac{\rho}{4}$ and disjoint from $\left[t_{i}, t_{i}+\right.$ $\left.\frac{\rho}{8}\right] \cup\left[t_{i+1}-\frac{\rho}{8}, t_{i+1}\right]$. We deduce from (5.18) that

$$
\int_{E_{i}^{\prime \prime}}|z|^{p} \geq \text { const. } \int_{\frac{\rho}{8}}^{\frac{\rho}{4}}\left(\frac{\bar{V}}{2} s\right)^{\frac{p}{2}} \mathrm{~d} s \geq \text { const. } \bar{V}^{\frac{p}{2}} \geq \text { const. }\left|E_{i}\right| \bar{V}^{\frac{p}{2}} .
$$

The author would like to thank E.D. Sontag for suggesting the inequality given in equation (1.9). 


\section{REFERENCES}

[1] V. Blondel, E. Sontag, M. Vidyasagar and J. Willems, Open Problems in Mathematical Systems and Control Theory. SpringerVerlag, London (1999).

[2] J.C. Doyle, T.T. Georgiou and M.C. Smith, The parallel projection operators of a nonlinear feedback system, in Proc. $31^{s t}$ IEEE Conf. Dec and Control. Tucson, AZ, IEEE Publications, Piscataway, NJ (1992) 1050-1054.

[3] A.T. Fuller, In the large stability of relay and saturated control with linear controllers. Internat. J. Control 10 (1969) 457-480.

[4] D.J. Hill, Dissipative nonlinear systems: Basic properties and stability analysis, in Proc. $31^{\text {st }}$ IEEE Conf. Dec and Control. Tucson, AZ, IEEE Publications, Piscataway, NJ (1992) 3259-3264.

[5] W. Liu, Y. Chitour and E.D. Sontag, On finite-gain stabilization of linear systems subject to input saturation. SIAM J. Control Optim. 4 (1996) 1190-1219.

[6] Z. Lin and A. Saberi, A semi-global low and high gain design technique for linear systems with input saturation stabilization and disturbance rejection. Internat. J. Robust Nonlinear Control 5 (1995) 381-398.

[7] Z. Lin, A. Saberi and A.R. Teel, Simultaneous $L^{p}$-stabilization and internal stabilization of linear systems subject to input saturation - state feedback case. Systems Control Lett. 25 (1995) 219-226.

[8] A. Megretsky, A gain scheduled for systems with saturation which makes the closed loop system $L^{2}$-bounded. Preprint (1996).

[9] E.D. Sontag, Mathematical theory of control. Springer-Verlag, New York (1990).

[10] E.D. Sontag, Stability and stabilization: Discontinuities and the effect of disturbances, in Nonlinear analysis, differential equation and control, edited by F.H. Clarke and R.J. Stern, Nato Sciences Series C 528 (1999).

[11] E.D. Sontag and H.J. Sussmann, Remarks on continuous feedbacks, in Proc. IEEE Conf. Dec and Control. Albuquerque, IEEE Publications, Piscataway, NJ (1980) 916-921.

[12] A.R. Teel, Global Stabilization and restricted tracking for multiple integrators with bounded controls. Systems Control Lett. 24 (1992) 165-171.

[13] Y. Yang, H.J. Sussmann and E.D. Sontag, Stabilization of linear systems with bounded controls. IEEE Trans. Automat. Control 39 (1994) 2411-2425.

[14] Y. Yang, Global stabilization of linear systems with bounded feedbacks. Ph.D. Thesis, Rutgers University (1993).

[15] A.J. Van Der Schaft, $L^{2}$-gain and passivity techniques in nonlinear control. Springer, London, Lecture Notes in Control and Inform. Sci. (1996). 